Article

\title{
Multi-Attribute Decision Making Method Based on Neutrosophic Vague $N$-Soft Sets
}

\author{
Jianbo Liu, Yanan Chen *, Ziyue Chen and Yanyan Zhang \\ School of Mathematics and Statistics, Northeastern University at Qinhuangdao, Qinhuangdao 066004, China; \\ jbliu@neuq.edu.cn (J.L.); ziyuechen1996@163.com (Z.C.); zhangyy@neuq.edu.cn (Y.Z.) \\ * Correspondence: chenyn0515@163.com; Tel.: +86-1873-350-9262
}

Received: 21 March 2020; Accepted: 11 May 2020; Published: 22 May 2020

check for

updates

\begin{abstract}
This paper proposes neutrosophic vague $N$-soft sets which is composed of neutrosophic vague sets and $N$-soft sets for the first time. The new hybrid model includes a pair of asymmetric functions: truth-membership and false-membership, and an indeterminacy-membership function. Some useful operations and propositions are given and illustrated by examples. Moreover, a method of priority relation ranking based on neutrosophic vague $N$-soft sets is presented. The validity of the method is verified by comparison. It is more flexible and reasonable to use this method in our daily life. Finally, a potential application of multi-attribute decision making is presented.
\end{abstract}

Keywords: neutrosophic vague sets; $N$-soft sets; priority relation ranking method; multi-attribute decision making

\section{Introduction}

Life is full of uncertainty, and people's cognition begins with uncertainty. By dealing with uncertain information, we can obtain more objective judgment. A lot of scholars in related fields have put forward some theories to solve these problems involving vagueness or subjectivity, such as fuzzy sets [1], rough sets [2], intuitionistic fuzzy sets [3], vague sets [4] and so on. But these theories still have many shortcomings in some problems, Molodtsov [5] thought that one reason for these shortcomings may be the lack of the parametrization tool of the theory. Subsequently the soft set theory overcame these shortcomings, which was introduced by Molodtsov [5] in 1999. Then, there are many mixed models of the soft sets. Maji proposed the fuzzy soft sets [6] and the intuitionistic fuzzy soft sets [7]. Yang [8] proposed the interval fuzzy soft sets. Xu [9] proposed vague soft sets. The concept of possibility fuzzy soft sets as extensions of soft set was proposed by Alkhazaleh et al. [10]. Feng et al. [11] proposed the basic concepts of rough fuzzy sets, rough soft sets, soft rough sets and soft rough fuzzy sets through fusion fuzzy sets, and gave corresponding basic properties. Peng [12] proposed interval valued intuitionistic hesitant fuzzy soft sets.

In terms of uncertainty, the fuzzy sets and the intuitionistic fuzzy sets are not as good as the neutrosophic sets. As an extension of the intuitionistic fuzzy sets, neutrosophic set [13] was introduced by Smarandache. Then Maji [14] introduced neutrosophic soft sets in 2013. The theory of neutrosophic vague set was proposed by Alkhazaleh [15] in 2015. Moreover, there are many hybrid models of neutrosophic sets, such as intuitionistic neutrosophic soft sets [16], interval-valued neutrosophic soft sets [17], neutrosophic vague soft sets [18] and so on. Of course, there are many methods to be applied in neutrosophic sets and its hybrid models, such as similarity measures between interval neutrosophic sets [19], similarity and entropy of neutrosophic soft sets [20], improved correlation coefficients of neutrosophic sets [21] and so on.

From the hybrid models of soft sets, most researchers use binary evaluation as usual. However, in our daily life, we often find non-binary data. An extended model of soft sets called $N$-soft sets was 
designed by Fatimah et al. [22]. It describes the importance of order grades in practical problems, breaks away from binary constraints and opens up our thoughts. However, $\mathrm{N}$-soft set is not enough to express content about rating or grades occurrence, nor can it specifically describe the occurrence of vagueness. For this purpose, as generalized models of $N$-soft sets, fuzzy $N$-soft sets, hesitant $N$-soft sets, hesitant fuzzy $N$-soft sets, interval valued hesitant fuzzy $N$-soft sets and intuitionistic fuzzy $N$-soft rough sets were introduced by Akram et al. [23-27].

Multi-attribute decision making refers to the process of finding the optimal solution by comprehensively evaluating multiple standard values of all solutions in a complex scenario. Fatimah et al. [22] use $N$-sets choice values and $T$ - choice values to rank the alternatives in $U$. The decision process is an extension of the decision process of the soft set without reduction of the parameters. Akram et al. [23] used score function method and comparison table method to solve practical problems about $(F, N)$-soft sets. And Akram et al. also extended the Technique for Order Preference by Similarity to an Ideal Solution (TOPSIS) method to deal with complex and interval-valued hesitant fuzzy $N$-soft sets information. Harish Garg et al. [28] proposed a multi-criteria decision method about single-valued neutrosophic sets based on frank choquet heronian mean operator. Peide Liu et al. [29] proposed a new three-way decision model with intuitionistic fuzzy numbers to solve multiple attribute decision making problems. Ordinal Priority Approach (OPA) in multiple attribute decision making [30] is designed to solve all types of multi-attribute decision making problems including group decision making and obtaining weights of experts. Ordered weighted averaging distance measure based on the single-valued neutrosophic linguistic was further presented in [31]. At present, more and more researchers are working on a better multi-attribute decision making method.

In the paper, we introduce a new concept called neutrosophic vague $N$-soft sets based on both neutrosophic vague soft sets and $N$-soft sets. The new hybrid model includes a pair of asymmetric functions: truth-membership and false-membership, and an indeterminacy-membership function. We use $\delta_{k p}(x)$ to define the grade of object $x_{i}$ under attribute $e_{j}$, where $k$-degree risk value represents the risk preference of the decision maker and $k$ may be a number between 0 and 1 or a closed sub-interval of $[0,1]$. In the definition of grade, $\delta_{k p}(x)$ containing the idea of probability should be a variable based on the ratio of truth membership $\hat{T}_{N V}(x)$ and false membership $\hat{F}_{N V}(x)$. Therefore, this model can make a very flexible decision based on the opinions of decision maker and experts. What's more, priority relation ranking method based on neutrosophic vague $N$-soft sets is proposed for the first time. It can not only ensure that the research object can meet the decision maker's requirements under each attribute, but also effectively avoid that the value of the good and bad attributes to be canceled out each other. By comparing the ranking results of the existing methods and our proposed method, we observe that their result is the same, which means that there is a consistency among our proposed decision making method and the existing methods. But the method we proposed is more reasonable because the idea of probability is implied in the grade. So the method is more reasonable and effective when considering the overall situation. The comparison of the proposed concept with some other concepts is denoted by Table 1 .

Table 1. Comparison of the proposed concept with some other concepts.

\begin{tabular}{ccccc}
\hline $\begin{array}{c}\text { The References } \\
\text { of Theories }\end{array}$ & $\begin{array}{c}\text { Parametrization } \\
\text { Tool }\end{array}$ & N-Binary & $\begin{array}{c}\text { Contains Information about } \\
\text { the Occurrence of Grades }\end{array}$ & $\begin{array}{c}\text { More Reasonable } \\
\text { Grade Definition }\end{array}$ \\
\hline$[1-4]$ etc. & inadequacy & - & - & - \\
{$[5-12,16-18]$ etc. } & adequacy & binary & - & - \\
{$[22]$} & adequacy & $N$-binary & No & No \\
{$[23-27]$ etc. } & adequacy & $N$-binary & Yes & Yes \\
\hline
\end{tabular}

The organization of this paper is as follows. Some new definitions related to newly proposed model were provided in Section 2. In Section 3, definitions, basic operations and propositions about neutrosophic vague $\mathrm{N}$-soft sets are introduced. In Section 4 , the priority relation ranking method 
based on neutrosophic vague $N$-soft sets is proposed and it is applied to practical problems. Finally, conclusion is presented in Section 5.

\section{Preliminaries}

In this section, in order to complete the presentation and facilitate future discussion, we will review several definitions that are useful for our paper.

Definition 1. [4] Let $U=\left\{x_{1}, x_{2}, \cdots, x_{n}\right\}$. A vague set $A=\left\{x_{i},\left[t_{A}\left(x_{i}\right), 1-f_{A}\left(x_{i}\right)\right] \mid x_{i} \in U\right\}$, i.e., $A\left(x_{i}\right)=\left[t_{A}\left(x_{i}\right), 1-f_{A}\left(x_{i}\right)\right]$ and the condition $0 \leq t_{A}\left(x_{i}\right) \leq 1-f_{A}\left(x_{i}\right)$ should hold for any $x_{i} \in U$, where $t_{A}\left(x_{i}\right)$ is called the true membership degree of element $x_{i}$ to the vague set $A$, while $f_{A}\left(x_{i}\right)$ is the degree of false membership of the element $x_{i}$ to the vague set $A$.

Definition 2. [4] Let $U=\left\{x_{1}, x_{2}, \cdots, x_{n}\right\}, A, B$ be two vague sets, then their union, intersection, and the complement of vague sets $A$ are defined as follows:

$$
\begin{gathered}
A \cup B=\left\{\left(x_{i},\left[\max \left(t_{A}\left(x_{i}\right), t_{B}\left(x_{i}\right)\right), \max \left(1-f_{A}\left(x_{i}\right), 1-f_{B}\left(x_{i}\right)\right)\right] \mid x_{i} \in U\right)\right\}, \\
A \cap B=\left\{\left(x_{i},\left[\min \left(t_{A}\left(x_{i}\right), t_{B}\left(x_{i}\right)\right), \min \left(1-f_{A}\left(x_{i}\right), 1-f_{B}\left(x_{i}\right)\right)\right] \mid x_{i} \in U\right)\right\}, \\
A^{c}=\left\{\left(x_{i},\left[f_{A}\left(x_{i}\right), 1-t_{A}\left(x_{i}\right)\right] \mid x_{i} \in U\right)\right\} .
\end{gathered}
$$

Definition 3. [4] Let $U=\left\{x_{1}, x_{2}, \cdots, x_{n}\right\}, A, B$ be two vague sets. If $\forall x_{i} \in U, t_{A}\left(x_{i}\right) \leq t_{B}\left(x_{i}\right), 1-$ $f_{A}\left(x_{i}\right) \leq 1-f_{B}\left(x_{i}\right)$, then $A$ is called a vague subset of $B$, denoted by $A \subseteq B$, where $1 \leq i \leq n$.

Definition 4. [13] Let $U$ be a universe of objects. A neutrosophic set $A_{N}$ in $U$ is characterized by a truth-membership function $T_{A_{N}}$ a indeterminacy-membership function $I_{A_{N}}$ and a falsity-membership function $F_{A_{N}} . T_{A_{N}}(x), I_{A_{N}}(x)$ and $F_{A_{N}}(x)$ are real standard or nonstandard subsets of $]^{-} 0,1^{+}[$. It can be written as $\left.A_{N}=\left\{<x,\left(T_{A_{N}}(x), I_{A_{N}}(x), F_{A_{N}}(x)\right)\right\rangle: x \in U\right\}$. There is no restriction on the sum of $T_{A_{N}}(x), I_{A_{N}}(x)$ and $F_{A_{N}}(x)$, so- $0 \leq \operatorname{supT}_{A_{N}}(x)+\operatorname{supI}_{A_{N}}(x)+\operatorname{supF}_{A_{N}}(x) \leq 3^{+}$.

Here, $1^{+}=1+\varepsilon$, where 1 is its standard part and $\varepsilon$ its non-standard part. Similarly, ${ }^{-} 0=0-\varepsilon$, where 0 is its standard part and $\varepsilon$ its non-standard part. However, $]^{-} 0,1^{+}$[ will be difficult to be applied in the real applications, so we need to replace $]^{-} 0,1^{+}[$with the interval $[0,1]$ in practical applications. We can see single valued neutrosphic sets in [32].

Definition 5. [15] Let $A_{N V}$ is a neutrosophic vague set on the universe of $U$ and $A_{N V}=\left\{<x ; \hat{T}_{A_{N V}}(x)\right.$; $\left.\hat{I}_{A_{N V}}(x) ; \hat{F}_{A_{N V}}(x) ; x \in U>\right\}$ whose truth-membership, indeterminacy-membership, and falsity-membership functions are defined as follows:

$$
\begin{gathered}
\hat{T}_{A_{N V}}(x)=\left[T_{A_{N V}}^{-}, T_{A_{N V}}^{+}\right]=\left[T_{A_{N V}}^{-} 1-F_{A_{N V}}^{-}\right], \hat{I}_{A_{N V}}(x)=\left[I_{A_{N V}}^{-} I_{A_{N V}}^{+}\right], \\
\hat{F}_{A_{N V}}(x)=\left[F_{A_{N V}}^{-}, F_{A_{N V}}^{+}\right]=\left[F_{A_{N V}}^{-}, 1-T_{A_{N V}}^{-}\right], \text {where } 0 \leq T_{A_{N V}}^{-}+I_{A_{N V}}^{-}+F_{A_{N V}}^{-} \leq 2^{+} .
\end{gathered}
$$

Definition 6. [15] Let $A_{N V}$ and $B_{N V}$ be two neutrosophic vague sets in the universe $U$, then the intersection $H_{N V}=A_{N V} \cap B_{N V}$, union $C_{N V}=A_{N V} \cup B_{N V}$ and the complement of vague sets $A_{N V}^{c}$ are defined as follows:

$$
H_{N V}=\left\{<x ; \hat{T}_{H_{N V}}(x) ; \hat{I}_{H_{N V}}(x) ; \hat{F}_{H_{N V}}(x) ; x \in U>\right\},
$$

where $\hat{T}_{H_{N V}}(x)=\left[\min \left(T_{A_{N V}}^{-}, T_{B_{N V}}^{-}\right), \min \left(T_{A_{N V}}^{+}, T_{B_{N V}}^{+}\right)\right], \quad \hat{I}_{H_{N V}}(x)=\left[\max \left(I_{A_{N V}}^{-}, I_{B_{N V}}^{-}\right), \max \left(I_{A_{N V}}^{+}, I_{B_{N V}}^{+}\right)\right]$, $\hat{F}_{H_{N V}}(x)=\left[\max \left(F_{A_{N V}}^{-}, F_{B_{N V}}^{-}\right), \max \left(F_{A_{N V}}^{+}, F_{B_{N V}}^{+}\right)\right]$;

$$
C_{N V}=\left\{<x ; \hat{T}_{C_{N V}}(x) ; \hat{I}_{C_{N V}}(x) ; \hat{F}_{C_{N V}}(x) ; x \in U>\right\},
$$

where $\hat{T}_{C_{N V}}(x)=\left[\max \left(T_{A_{N V}}^{-}, T_{B_{N V}}^{-}\right), \max \left(T_{A_{N V}}^{+}, T_{B_{N V}}^{+}\right)\right], \quad \hat{I}_{C_{N V}}(x)=\left[\min \left(I_{A_{N V}}^{-} I_{B_{N V}}^{-}\right), \min \left(I_{A_{N V}}^{+}, I_{B_{N V}}^{+}\right)\right]$, $\hat{F}_{C_{N V}}(x)=\left[\min \left(F_{A_{N V}}^{-}, F_{B_{N V}}^{-}\right), \min \left(F_{A_{N V}}^{+}, F_{B_{N V}}^{+}\right)\right]$;

$$
A_{N V}^{c}=\left\{<x ; \hat{T}_{A_{N V}}^{c}(x) ; \hat{I}_{A_{N V_{x}}^{c}}^{c}(x) ; \hat{F}_{A_{N V_{x}}^{c}}^{c}(x) ; x \in U>\right\},
$$


where $\hat{T}_{A_{N V}}^{c}(x)=\left[1-T_{A_{N V}}^{+}, 1-T_{A_{N V}}^{-}\right], \hat{I}_{A_{N V x}}^{c}(x)=\left[1-I_{A_{N V}}^{+}, 1-I_{A_{N V}}^{-}\right], \hat{F}_{A_{N V}}^{c}(x)=\left[1-F_{A_{N V}}^{+}, 1-F_{A_{N V}}^{-}\right]$.

Definition 7. [18] Let $U$ be a universe, $E$ a set of parameters and $T \subseteq E .(\hat{F}, T)$ is called a neutrosophic vague soft set (NVSS) over $U$ where $\hat{F}$ is a mapping given by $\hat{F}: T \rightarrow N V(U)$, and $N V(U)$ denotes the set of all neutrosophic vague subsets of $U$.

Definition 8. [22] Let $U$ be a universe under consideration and $E$ be a set of attributes, $T \subseteq E$. Let $G=$ $\{0,1,2, \cdots, N-1\}$ be a set of ordered grades, where $N \in\{2,3, \cdots\}$. A triple $(F, T, N)$ is called an $N$-soft set on $U$ if $F$ is a mapping from $T$ to $2^{U \times G}$, for each $t \in T$ and $x \in U$, there is a unique $\left(x, g_{t}\right) \in U \times G$ such that $\left(x, g_{t}\right) \in F(t), g_{t} \in G$.

\section{Neutrosophic Vague N-Soft Sets}

\subsection{The Concept of Neutrosophic Vague N-Soft Sets}

If $U=\left\{x_{1}, x_{2}, \cdots, x_{n}\right\}$ and $E=\left\{e_{1}, e_{2}, \cdots, e_{m}\right\}$. We denote by $N V(E)$ the set of all neutrosophic vague soft sets on $U$, then we describe the mapping $N V$ as :

$$
\begin{aligned}
N V\left(e_{j}\right)= & \left\{<x_{1}, \hat{T}_{N V}\left(x_{1}\right), \hat{I}_{N V}\left(x_{1}\right), \hat{F}_{N V}\left(x_{1}\right)>, \ldots,<x_{n}, \hat{T}_{N V}\left(x_{n}\right), \hat{I}_{N V}\left(x_{n}\right), \hat{F}_{N V}\left(x_{n}\right)>\right\} \\
= & \left\{<x_{1},\left[T_{N V}^{-}\left(x_{1}\right), T_{N V}^{+}\left(x_{1}\right)\right],\left[I_{N V}^{-}\left(x_{1}\right), I_{N V}^{+}\left(x_{1}\right)\right],\left[F_{N V}^{-}\left(x_{1}\right), F_{N V}^{+}\left(x_{1}\right)\right]>, \cdots,\right. \\
& \left.<x_{n},\left[T_{N V}^{-}\left(x_{n}\right), T_{N V}^{+}\left(x_{n}\right)\right],\left[I_{N V}^{-}\left(x_{n}\right), I_{N V}^{+}\left(x_{n}\right)\right],\left[F_{N V}^{-}\left(x_{n}\right), F_{N V}^{+}\left(x_{n}\right)\right]>\right\}
\end{aligned}
$$

where $e_{j} \in E, x_{i} \in U, T_{N V}^{+}\left(x_{i}\right)=1-F_{N V}^{-}\left(x_{i}\right), F_{N V}^{+}\left(x_{i}\right)=1-T_{N V}^{-}\left(x_{i}\right),{ }^{-} 0 \leq T_{N V}^{-}\left(x_{i}\right)+I_{N V}^{-}\left(x_{i}\right)+$ $F_{N V}^{-}\left(x_{i}\right) \leq 2^{+} . \hat{T}_{N V}\left(x_{i}\right), \hat{I}_{N V}\left(x_{i}\right), \hat{F}_{N V}\left(x_{i}\right) \subseteq[0,1], i=1,2, \cdots, n ; j=1,2, \cdots, m$.

Definition 9. Let $U=\left\{x_{1}, \cdots, x_{i}, \cdots, x_{n}\right\}$ be a universe of objects under consideration and $E=$ $\left\{e_{1}, \cdots, e_{j}, \cdots, e_{m}\right\}$ be the universal set of parameters, $T \subseteq E$. A pair $(N V, K)$ is called a neutrosophic vague $N$-soft set (NVNSS), when $K=(F, T, N)$ is an $N$-soft set on $U$ with $N \in\{2,3, \cdots\}$ and a set of ordered grades $G=\{0,1, \cdots, N-1\} . N V$ is a mapping $N V: T \rightarrow \bigcup_{e_{j} \in T} \mathcal{N} \mathcal{V}\left(N V\left(e_{j}\right)\right), e_{j} \in T, i=1,2, \cdots, n$; $j=1,2, \cdots, m$.

For each $e_{j} \in T$ and , $x_{i} \in U$, there exists a unique $\left(x_{i}, g_{i j}\right) \in U \times G$ such that $g_{i j} \in G$. That is to say :

$$
\begin{gathered}
\mathcal{N V}\left(N V\left(e_{j}\right)\right)=\left\{<\left(x_{1}, g_{1 j}\right), \hat{T}_{N V}\left(x_{1}\right), \hat{I}_{N V}\left(x_{1}\right), \hat{F}_{N V}\left(x_{1}\right)>,\right. \\
\left.\cdots,<\left(x_{n}, g_{n j}\right), \hat{T}_{N V}\left(x_{n}\right), \hat{I}_{N V}\left(x_{n}\right), \hat{F}_{N V}\left(x_{n}\right)>\right\} .
\end{gathered}
$$

Neutrosophic vague $\mathrm{N}$-soft sets, combining the advantages of neutrosophic vague soft sets and $\mathrm{N}$-soft sets, are more accurate in dealing with vague and uncertain problems. If we only use the $\hat{T}_{N V}(x)$ to evaluated the research objects, we will ignore the effect of $\hat{I}_{N V}(x)$ and $\hat{F}_{N V}(x)$ in decision making problems. Hence the grading criteria followed by the membership $\hat{T}_{N V}(x), \hat{I}_{N V}(x)$ and $\hat{F}_{N V}(x)$ of research objects according to parameters.

Feng's expectation score function [33] is $\delta(A)=\frac{t_{A}-f_{A}+1}{2}=\frac{t_{A}+t_{A}+h_{A}}{2}=t_{A}+\frac{h_{A}}{2}$, so it aims to distribute the degree of hesitation equally to truth membership and false membership respectively. However, hesitation is not the same degree of support and opposition actually. In our daily life, we will encounter a lot of uncertain things. How to use these hesitant information efficiently to solve the problems is the key.

If we make decisions based on the opinions of everyone, we need to predict the hesitant parts, for which $\delta_{k p}(x)$ plays an important role. $\delta_{k p}(x)$ containing the idea of probability should be a variable based on the ratio of truth membership $\hat{T}_{N V}(x)$ and false membership $\hat{F}_{N V}(x)$. The following concept provides an updated version of Feng's expectation score function.

Definition 10. The expectation score function of neutrosophic vague soft sets based on probability $\delta_{k p}$ is a mapping. $\delta_{k p}: U \rightarrow[0,1]$. Here for each parameter e, we can get the grade by $\delta_{k p}(x)$ on the universe $U$. 


$$
\delta_{k p}(x)=\bar{\alpha}+\frac{\bar{\alpha}}{\bar{\alpha}+\bar{\gamma}} \cdot \bar{\beta}
$$

where $\alpha=T_{N V}^{-}(x)+\hat{k}\left(T_{N V}^{+}(x)-T_{N V}^{-}(x)\right), \beta=I_{N V}^{-}(x)+\hat{k}\left(I_{N V}^{+}(x)-I_{N V}^{-}(x)\right), \gamma=F_{N V}^{+}(x)-$ $\hat{k}\left(F_{N V}^{+}(x)-F_{N V}^{-}(x)\right), \bar{\alpha}=\frac{\alpha}{\alpha+\beta+\gamma}, \bar{\beta}=\frac{\beta}{\alpha+\beta+\gamma}, \bar{\gamma}=\frac{\gamma}{\alpha+\beta+\gamma}$, and satisfies $\hat{k} \in[0,1]$.

Remark 1. Here are a few explanations as follows:

1. $\delta_{k p}(x)$ contains the idea of probability, and forecasts the degree of support according to the ratio of support and opposition.

2. If the decision maker's risk attitude is not clear, then $k$-degree risk value is a interval value $\left[k^{-}, k^{+}\right]$. In general, $k$-degree risk value can be divided into the following two cases.

(1) If $k^{-}=k^{+}$, we think $\hat{k}=k^{-}=k^{+}$;

(2) If $k^{-} \neq k^{+}$, we think $\hat{k}=\sqrt{k^{-} \cdot k^{+}}$or $\hat{k}=\frac{k^{-}+k^{+}}{2}$.

3. In the definition of $\delta_{k p}(x), \alpha, \beta, \gamma$ represent $k$-degree risk value of the three interval values denoted by $\hat{T}_{N V}(x), \hat{I}_{N V}(x)$ and $\hat{F}_{N V}(x)$. Decision maker can choose the appropriate $k$-degree risk value according to actual situations, so it is more flexible and convenient to solve uncertain problems.

4. $\bar{\alpha}, \bar{\beta}, \bar{\gamma}$ are the standardized value of $\alpha, \beta, \gamma$ respectively. Hence $\delta_{k p}(x) \in[0,1]$.

Proposition 1. From the Definition 10, we can get the equation $\delta_{k p}(x)=\alpha$.

Proof. Since

$$
\begin{aligned}
\delta_{k p}(x) & =\bar{\alpha}+\frac{\bar{\alpha}}{\bar{\alpha}+\bar{\gamma}} \cdot \bar{\beta} \\
& =\frac{\alpha}{\alpha+\beta+\gamma}+\frac{\frac{\alpha}{\alpha+\beta+\gamma}}{\frac{\alpha}{\alpha+\beta+\gamma}+\frac{\gamma}{\alpha+\beta+\gamma}} \cdot \frac{\beta}{\alpha+\beta+\gamma} \\
& =\frac{\alpha}{\alpha+\beta+\gamma}+\frac{\alpha}{\alpha+\gamma} \cdot \frac{\beta}{\alpha+\beta+\gamma} \\
& =\frac{1}{\alpha+\beta+\gamma} \cdot\left(\alpha+\frac{\alpha \beta}{\alpha+\gamma}\right) \\
& =\frac{\alpha}{\alpha+\gamma} ;
\end{aligned}
$$

And then

$$
\begin{aligned}
\alpha+\gamma & =T_{N V}^{-}(x)+\hat{k}\left(T_{N V}^{+}(x)-T_{N V}^{-}(x)\right)+F_{N V}^{+}(x)-\hat{k}\left(F_{N V}^{+}\left(x_{i j}\right)-F_{N V}^{-}(x)\right) \\
& =T_{N V}^{-}(x)+\hat{k}\left(1-F_{N V}^{-}(x)-T_{N V}^{-}(x)\right)+1-T_{N V}^{-}(x)-\hat{k}\left(1-T_{N V}^{-}(x)-F_{N V}^{-}(x)\right) \\
& =1
\end{aligned}
$$

So $\delta_{k p}(x)=\alpha$, where $T_{N V}^{+}(x)=1-F_{N V}^{-}(x), F_{N V}^{+}(x)=1-T_{N V}^{-}(x)$.

Of course, the decision maker can follow this criteria on the basis of different objects under different attributes to give the grades:

$$
\begin{aligned}
& 0.0 \leq \delta_{k p}(x)<0.1, \text { when } g=0 ; \\
& 0.1 \leq \delta_{k p}(x)<0.3, \text { when } g=1 ; \\
& 0.3 \leq \delta_{k p}(x)<0.5, \text { when } g=2 ; \\
& 0.5 \leq \delta_{k p}(x)<0.8, \text { when } g=3 ; \\
& 0.8 \leq \delta_{k p}(x) \leq 1.0, \text { when } g=4 .
\end{aligned}
$$


Remark 2. Here are a few instructions as follows:

1. Any NV2SS can be naturally associated with a neutrosophic vague soft set. We define a NV2SS, $f: E \rightarrow$ $N V^{(U \times(0,1))}$ with a $N V(\sigma, E)$, so for every $e_{j} \in E$, we can get

$$
\sigma\left(e_{j}\right)=\left\{\left(x, \hat{T}_{N V}(x), \hat{I}_{N V}(x), \hat{F}_{N V}(x)\right) \mid<(x, 1), \hat{T}_{N V}(x), \hat{I}_{N V}(x), \hat{F}_{N V}(x)>\in f\left(e_{j}\right)\right\} .
$$

2. Any NV2SS on a universe $U$ can be taken as an NVN*SS with $N^{*}>N$ arbitrary. That is to say that the grade $N^{*}$ exists, but it's never be used.

3. Grade $0 \in G$ represents the lowest grade. It doesn't mean that there is incomplete information.

Example 1. Let $U=\left\{x_{1}, x_{2}, x_{3}\right\}$ represents different films and $T=\left\{e_{1}, e_{2}\right\}=\{$ actor, type $\}$ be the set of parameters. If we use a 0.5-degree risk value $(\hat{k}=0.5)$, a neutrosophic vague 4 -soft set $\left(N V_{1}, K_{1}\right)$ can be obtained from Table 2 easily.

Table 2. The $\left(N V_{1}, K_{1}\right)$ in Example 1.

\begin{tabular}{ccc}
\hline$\left(N V_{\mathbf{1}}, \boldsymbol{K}_{\mathbf{1}}\right)$ & $\boldsymbol{e}_{\mathbf{1}}$ & $\boldsymbol{e}_{\mathbf{2}}$ \\
\hline$x_{1}$ & $<3,[0.20,0.80],[0.10,0.90],[0.20,0.80]>$ & $<1,[0.10,0.30],[0.50,0.70],[0.70,0.90]>$ \\
$x_{2}$ & $<3,[0.30,0.90],[0.30,0.50],[0.10,0.70]>$ & $<3,[0.40,0.80],[0.30,0.60],[0.20,0.60]>$ \\
$x_{3}$ & $<2,[0.40,0.50],[0.30,0.50],[0.50,0.60]>$ & $<2,[0.20,0.70],[0.20,0.50],[0.30,0.80]>$ \\
\hline
\end{tabular}

\subsection{The Operations and Propositions of NVNSSs}

Definition 11. Let $\left(N V_{1}, K_{1}\right)$ and $\left(N V_{2}, K_{2}\right)$ be two NVNSSs on a universe $U$, where $K_{1}=\left(N V_{1}, T, N_{1}\right)$ and $K_{2}=\left(N V_{2}, S, N_{2}\right)$ are $N$-soft sets at the same $k$-degree risk value. $T \subseteq E, S \subseteq E$. If $\forall x \in U$,

(1) $T \subseteq S$;

(2) $g_{1} \leq g_{2}$;

(3) $N V_{1}$ is also a neutrosophic vague soft subset of $N V_{2}$.

That is to say

$$
\begin{gathered}
\hat{T}_{N V_{1}}(x) \leq \hat{T}_{N V_{2}}(x), \hat{I}_{N V_{1}}(x) \geq \hat{I}_{N V_{2}}(x), \hat{F}_{N V_{1}}(x) \geq \hat{F}_{N V_{2}}(x) \Leftrightarrow T_{N V_{1}}^{-}(x) \leq T_{N V_{2}}^{-}(x), T_{N V_{1}}^{+}(x) \leq T_{N V_{2}}^{+}(x) ; \\
I_{N V_{1}}^{-}(x) \geq I_{N V_{2}}^{+}(x), I_{N V_{1}}^{+}(x) \geq I_{N V_{2}}^{-}(x) ; F_{N V_{1}}^{-}(x) \geq F_{N V_{2}}^{-}(x), F_{N V_{1}}^{+}(x) \geq F_{N V_{2}}^{+}(x) .
\end{gathered}
$$

Then $\left(N V_{1}, K_{1}\right)$ is said to be a neutrosophic vague $N$-soft subset of $\left(N V_{2}, K_{2}\right)$, denoted by $\left(N V_{1}, K_{1}\right) \widetilde{\subseteq}$ $\left(N V_{2}, K_{2}\right)$.

Example 2. Let $\left(N V_{2}, K_{2}\right)$ be a neutrosophic vague 5-soft set in Table 3. $S \subseteq E$ and $S=\left\{e_{1}, e_{2}, e_{3}, e_{4}\right\}=$ $\{$ actor, type, advertisement, social meaning $\}$. If we use a 0.5 -degree risk value $(\hat{k}=0.5)$, then we can find $\left(N V_{1}\right.$, $\left.K_{1}\right) \widetilde{\subseteq}\left(N V_{2}, K_{2}\right)$

Definition 12. Let $\left(N V_{1}, K_{1}\right)$ and $\left(N V_{2}, K_{2}\right)$ be two $N V N S S s$ on a universe $U$, where $K_{1}=\left(N V_{1}, T, N_{1}\right)$ and $K_{2}=\left(N V_{2}, S, N_{2}\right)$ are $N$-soft sets at the same $k$-degree risk value. Then $\left(N V_{1}, K_{1}\right)$ and $\left(N V_{2}, K_{2}\right)$ are said to be equal if and only if $\left(N V_{1}, K_{1}\right) \widetilde{\subseteq}\left(N V_{2}, K_{2}\right)$ and $\left(N V_{2}, K_{2}\right) \widetilde{\subseteq}\left(N V_{1}, K_{1}\right)$. 
Table 3. The $\left(N V_{2}, K_{2}\right)$ in Example 2.

\begin{tabular}{ccc}
\hline$\left(N V_{2}, K_{2}\right)$ & $e_{1}$ & $e_{2}$ \\
\hline$x_{1}$ & $<3,[0.30,0.90],[0.10,0.70],[0.10,0.70]>$ & $<2,[0.20,0.40],[0.50,0.60],[0.60,0.80]>$ \\
$x_{2}$ & $<3,[0.40,0.80],[0.20,0.40],[0.10,0.60]>$ & $<3,[0.50,0.90],[0.30,0.50],[0.10,0.50]>$ \\
$x_{3}$ & $<3,[0.50,0.80],[0.20,0.30],[0.20,0.50]>$ & $<3,[0.40,0.70],[0.20,0.30],[0.30,0.60]>$ \\
\hline$\left(N V_{2}, K_{2}\right)$ & $e_{3}$ & $e_{4}$ \\
\hline$x_{1}$ & $<3,[0.50,0.80],[0.10,0.90],[0.20,0.50]>$ & $<4,[0.80,0.90],[0.50,0.70],[0.10,0.20]>$ \\
$x_{2}$ & $<4,[0.70,0.90],[0.30,0.50],[0.10,0.30]>$ & $<4,[0.70,0.90],[0.30,0.60],[0.10,0.30]>$ \\
$x_{3}$ & $<4,[0.80,0.90],[0.30,0.50],[0.10,0.20]>$ & $<3,[0.60,0.80],[0.20,0.50],[0.20,0.40]>$ \\
\hline
\end{tabular}

Definition 13. $A(N V, K)$-soft set is a neutrosophic null vague $N$-soft set denoted by $\varnothing_{N V}$. And $\varnothing_{N V}\left(e_{j}\right)=$ $\left\{<\left(x_{1}, 0\right),[0,0],[1,1],[1,1]>, \cdots,<\left(x_{n}, 0\right),[0,0],[1,1],[1,1]>\right\}$, where $\forall e_{j} \in T \subseteq E$.

Definition 14. $A(N V, K)$-soft set is a neutrosophic absolute vague $N$-soft set denoted by $A_{N V} . A_{N V}\left(e_{j}\right)=$ $\left\{<\left(o_{1}, N-1\right),[1,1],[0,0],[0,0]>, \cdots,<\left(o_{n}, N-1\right),[1,1],[0,0],[0,0]>\right\}$, where $\forall e_{j} \in T \subseteq E$.

Definition 15. Let $\left(N V_{1}, K_{1}\right)$ be a NVNSS. The complement of $\left(N V_{1}, K_{1}\right)$ is denoted by $\left(N V_{1}, K_{1}\right)^{c}$, if $\forall$ $e_{j} \in T$ and $x_{i} \in U, N_{1} \widetilde{\cap} N_{1}^{c}=\phi, N V_{1}^{c}$ is a neutrosophic vague $N$-soft complement of $N V_{1} . N V_{1}^{c}$ is defined by

$$
\begin{aligned}
\hat{T}_{N V_{1}}^{c}\left(x_{i}\right)= & {\left[1-T_{N V_{1}}^{+}\left(x_{i}\right), 1-T_{N V_{1}}^{-}\left(x_{i}\right)\right]=\left[F_{N V_{1}}^{-}\left(x_{i}\right), F_{N V_{1}}^{+}\left(x_{i}\right)\right], } \\
& \hat{I}_{N V_{1}}^{c}\left(x_{i}\right)=\left[1-I_{N V_{1}}^{+}\left(x_{i}\right), 1-I_{N V_{1}}^{-}\left(x_{i}\right)\right], \\
\hat{F}_{N V_{1}}^{c}\left(x_{i}\right)= & {\left[1-F_{N V_{1}}^{+}\left(x_{i}\right), 1-F_{N V_{1}}^{-}\left(x_{i}\right)\right]=\left[T_{N V_{1}}^{-}\left(x_{i}\right), T_{N V_{1}}^{+}\left(x_{i}\right)\right] . }
\end{aligned}
$$

Obviously, $\left(\left(N V_{1}, K_{1}\right)^{c}\right)^{c} \neq\left(N V_{1}, K_{1}\right)$ as the complements of $N$-soft set $\left(K_{1}^{c}\right)$ are not the only one, so we can get many complements of $\left(N V_{1}, K_{1}\right)$.

Example 3. $\left(N V_{1}, K_{1}\right)^{c}$ is one of the complements of $\left(N V_{1}, K_{1}\right)$, and it can be obtained in Table 4.

Table 4. One of the complements of $\left(N V_{1}, K_{1}\right)$ in Example 3.

\begin{tabular}{ccc}
\hline$\left(N V_{\mathbf{1}}, \boldsymbol{K}_{\mathbf{1}}\right)^{c}$ & $\boldsymbol{e}_{\mathbf{1}}$ & $\boldsymbol{e}_{\mathbf{2}}$ \\
\hline$x_{1}$ & $<2,[0.20,0.80],[0.10,0.90],[0.20,0.80]>$ & $<3,[0.70,0.90],[0.30,0.50],[0.10,0.30]>$ \\
$x_{2}$ & $<2,[0.10,0.70],[0.50,0.70],[0.30,0.90]>$ & $<2,[0.20,0.60],[0.40,0.70],[0.40,0.80]>$ \\
$x_{3}$ & $<3,[0.50,0.60],[0.50,0.70],[0.40,0.50]>$ & $<3,[0.30,0.80],[0.50,0.80],[0.20,0.70]>$ \\
\hline
\end{tabular}

Definition 16. Let $U$ be a universe, and let $\left(N V_{1}, K_{1}\right)$ and $\left(N V_{2}, K_{2}\right)$ be two NVNSSs, $K_{1}=\left(N V_{1}, T, N_{1}\right)$ and $K_{2}=\left(N V_{2}, S, N_{2}\right)$ are $N$-soft sets on $U$ at the same $k$-degree risk value. So their restricted intersection is denoted by $\left(N V_{1}, K_{1}\right) \widetilde{\cap}_{R}\left(N V_{2}, K_{2}\right)$ and it is defined as $\left(\eta_{N V}, K_{1} \cap_{\mathcal{R}} K_{2}\right)$, where $K_{1} \cap_{\mathcal{R}} K_{2}=(E, T \cap$ $\left.S, \min \left(N_{1}, N_{2}\right)\right), \forall e_{j} \in T \cap S$ and $x_{i} \in U,<\left(x_{i}, g_{i j}\right), \hat{T}_{N V}\left(x_{i}\right), \hat{I}_{N V}\left(x_{i}\right), \hat{F}_{N V}\left(x_{i}\right)>\in \eta_{N V}\left(e_{j}\right) \Leftrightarrow$

$$
\begin{gathered}
g_{i j}=\min \left(g_{i j}^{1}, g_{i j}^{2}\right), \\
\hat{T}_{N V}\left(x_{i}\right)=\left[\min \left(T_{N V_{1}}^{-}\left(x_{i}\right), T_{N V_{2}}^{-}\left(x_{i}\right)\right), \min \left(T_{N V_{1}}^{+}\left(x_{i}\right), T_{N V_{2}}^{+}\left(x_{i}\right)\right)\right], \\
\hat{I}_{N V}\left(x_{i}\right)=\left[\max \left(I_{N V_{1}}^{-}\left(x_{i}\right), I_{N V_{2}}^{-}\left(x_{i}\right)\right), \max \left(I_{N V_{1}}^{+}\left(x_{i}\right), I_{N V_{2}}^{+}\left(x_{i}\right)\right)\right], \text { and } \\
\hat{F}_{N V}\left(x_{i}\right)=\left[\max \left(F_{N V_{1}}^{-}\left(x_{i}\right), F_{N V_{2}}^{-}\left(x_{i}\right)\right), \max \left(F_{N V_{1}}^{+}\left(x_{i}\right), F_{N V_{2}}^{+}\left(x_{i}\right)\right)\right] .
\end{gathered}
$$

Example 4. Let $U=\left\{x_{1}, x_{2}, x_{3}\right\}$ represents different films and $T=\left\{e_{1}, e_{2}\right\}=\{$ actor, type $\}$ be the set of parameters. If we use a 0.5 -degree risk value $(\hat{k}=0.5)$, a neutrosophic vague 4-soft set $\left(N V_{3}, K_{3}\right)$ can be obtained from Table 5 easily. So $\left(N V_{2}, K_{2}\right) \widetilde{\cap}_{R}\left(N V_{3}, K_{3}\right)$ can be obtained from Table 6. 
Table 5. The $\left(N V_{3}, K_{3}\right)$ in Example 4.

\begin{tabular}{ccc}
\hline$\left(N V_{3}, K_{3}\right)$ & $e_{1}$ & $e_{2}$ \\
\hline$x_{1}$ & $<3,[0.60,0.90],[0.10,0.40],[0.10,0.40]>$ & $<3,[0.40,0.70],[0.20,0.80],[0.30,0.60]>$ \\
$x_{2}$ & $<3,[0.70,0.80],[0.20,0.50],[0.20,0.30]>$ & $<4,[0.70,0.90],[0.20,0.30],[0.10,0.30]>$ \\
$x_{3}$ & $<2,[0.30,0.40],[0.10,0.40],[0.60,0.70]>$ & $<3,[0.60,0.70],[0.20,0.40],[0.30,0.40]>$ \\
\hline
\end{tabular}

Table 6. The $\left(N V_{2}, K_{2}\right) \widetilde{\cap}_{R}\left(N V_{3}, K_{3}\right)$ in Example 4.

\begin{tabular}{ccc}
\hline$\left(N V_{2}, K_{2}\right) \widetilde{\cap}_{R}\left(N V_{3}, K_{3}\right)$ & $e_{1}$ & $e_{2}$ \\
\hline$x_{1}$ & $<3,[0.30,0.90],[0.10,0.70],[0.10,0.70]>$ & $<2,[0.20,0.40],[0.50,0.80],[0.60,0.80]>$ \\
$x_{2}$ & $<3,[0.40,0.80],[0.20,0.50],[0.20,0.60]>$ & $<3,[0.50,0.90],[0.30,0.50],[0.10,0.50]>$ \\
$x_{3}$ & $<2,[0.30,0.40],[0.20,0.40],[0.60,0.70]>$ & $<3,[0.40,0.70],[0.20,0.40],[0.30,0.60]>$ \\
\hline
\end{tabular}

Definition 17. Let $U$ be universe of objects, and let $\left(N V_{1}, K_{1}\right)$ and $\left(N V_{2}, K_{2}\right)$ be two NVNSSs, $K_{1}=\left(N V_{1}, T, N_{1}\right)$ and $K_{2}=\left(N V_{2}, S, N_{2}\right)$ are $N$-soft sets on $U$ at the same $k$-degree risk value. Then their restricted union is denoted by $\left(N V_{1}, K_{1}\right) \widetilde{\cup}_{R}\left(N V_{2}, K_{2}\right)$ and it is defined as $\left(\zeta_{N V}, K_{1} \cup_{\mathcal{R}} K_{2}\right)$, where $K_{1} \cup_{\mathcal{R}} K_{2}=$ $\left(E, T \cap S, \max \left(N_{1}, N_{2}\right)\right), \forall e_{j} \in T \cap S$ and $x_{i} \in U,<\left(x_{i}, g_{i j}\right), \hat{T}_{N V}\left(x_{i}\right), \hat{I}_{N V}\left(x_{i}\right), \hat{F}_{N V}\left(x_{i}\right)>\in \zeta_{N V}\left(e_{j}\right) \Leftrightarrow$

$$
\begin{gathered}
g_{i j}=\max \left(g_{i j}^{1}, g_{i j}^{2}\right), \\
\hat{T}_{N V}\left(x_{i}\right)=\left[\max \left(T_{N V_{1}}^{-}\left(x_{i}\right), T_{N V_{2}}^{-}\left(x_{i}\right)\right), \max \left(T_{N V_{1}}^{+}\left(x_{i}\right), T_{N V_{2}}^{+}\left(x_{i}\right)\right)\right], \\
\hat{I}_{N V}\left(x_{i}\right)=\left[\min \left(I_{N V_{1}}^{-}\left(x_{i}\right), I_{N V_{2}}^{-}\left(x_{i}\right)\right), \min \left(I_{N V_{1}}^{+}\left(x_{i}\right), I_{N V_{2}}^{+}\left(x_{i}\right)\right)\right], \text { and } \\
\hat{F}_{N V}\left(x_{i}\right)=\left[\min \left(F_{N V_{1}}^{-}\left(x_{i}\right), F_{N V_{2}}^{-}\left(x_{i}\right)\right), \min \left(F_{N V_{1}}^{+}\left(x_{i}\right), F_{N V_{2}}^{+}\left(x_{i}\right)\right)\right] .
\end{gathered}
$$

Example 5. We consider the two neutrosophic vague 5 -soft sets $\left(N V_{2}, K_{2}\right)$ and $\left(N V_{3}, K_{3}\right)$ defined in Example 2 and 4 respectively. We know $\left(N_{2}, K_{2}\right) \widetilde{\cup}_{R}\left(N V_{3}, K_{3}\right)$ can be obtained from Table 7 easily.

Table 7. The $\left(N V_{2}, K_{2}\right) \widetilde{\cup}_{R}\left(N V_{3}, K_{3}\right)$ in Example 5.

\begin{tabular}{ccc}
\hline$\left(N V_{2}, K_{2}\right)$ & $e_{1}$ & $e_{2}$ \\
\hline$x_{1}$ & $<3,[0.60,0.90],[0.10,0.40],[0.10,0.40]>$ & $<3,[0.40,0.70],[0.20,0.60],[0.30,0.60]>$ \\
$x_{2}$ & $<3,[0.70,0.80],[0.20,0.40],[0.10,0.30]>$ & $<4,[0.70,0.90],[0.20,0.30],[0.10,0.30]>$ \\
$x_{3}$ & $<3,[0.50,0.80],[0.10,0.30],[0.20,0.50]>$ & $<3,[0.60,0.70],[0.20,0.30],[0.30,0.40]>$ \\
\hline
\end{tabular}

Definition 18. Let $U$ be universe of objects, and let $\left(N V_{1}, K_{1}\right)$ and $\left(N V_{2}, K_{2}\right)$ be two NVNSSs, $K_{1}=\left(N V_{1}, T, N_{1}\right)$ and $K_{2}=\left(N V_{2}, S, N_{2}\right)$ are $N$-soft sets on $U$ at the same $k$-degree risk value. Then their extended intersection is denoted by $\left(N V_{1}, K_{1}\right) \widetilde{\cap}_{E}\left(N V_{2}, K_{2}\right)$ and it is defined as $\left(\xi_{N V}, K_{1} \cap \mathcal{E} K_{2}\right)$, where $K_{1}$ $\cap_{\mathcal{E}} K_{2}=\left(B, T \cup S, \max \left(N_{1}, N_{2}\right)\right), \forall e_{j} \in T \cup S$ and $x_{i} \in U,<\left(x_{i}, g_{i j}\right), \hat{T}_{N V}\left(x_{i}\right), \hat{I}_{N V}\left(x_{i}\right), \hat{F}_{N V}\left(x_{i}\right)>\in$ $\xi_{N V}\left(e_{j}\right) \Leftrightarrow$

(1) $e_{j} \in T-S$ :

$$
\begin{gathered}
g_{i j}=g_{i j}^{1}, \hat{T}_{N V}\left(x_{i}\right)=\left[T_{N V_{1}}^{-}\left(x_{i}\right), T_{N V_{1}}^{+}\left(x_{i}\right)\right] \\
\hat{I}_{N V}\left(x_{i}\right)=\left[I_{N V_{1}}^{-}\left(x_{i}\right), I_{N V_{1}}^{+}\left(x_{i}\right)\right], \text { and } \hat{F}_{N V}\left(x_{i}\right)=\left[F_{N V_{1}}^{-}\left(x_{i}\right), F_{N V_{1}}^{+}\left(x_{i}\right)\right]
\end{gathered}
$$

(2) $e_{j} \in S-T$ :

$$
\begin{gathered}
g_{i j}=g_{i j}^{2}, \hat{T}_{N V}\left(x_{i}\right)=\left[T_{N V_{2}}^{-}\left(x_{i}\right), T_{N V_{2}}^{+}\left(x_{i}\right)\right] \\
\hat{I}_{N V}\left(x_{i}\right)=\left[I_{N V_{2}}^{-}\left(x_{i}\right), I_{N V_{2}}^{+}\left(x_{i}\right)\right], \text { and } \hat{F}_{N V}\left(x_{i}\right)=\left[F_{N V_{2}}^{-}\left(x_{i}\right), F_{N V_{2}}^{+}\left(x_{i}\right)\right] ;
\end{gathered}
$$


(3) $e_{j} \in T \cap S$ :

$$
\begin{gathered}
g_{i j}=\min \left(g_{i j}^{1}, g_{i j}^{2}\right), \\
\hat{T}_{N V}\left(x_{i}\right)=\left[\min \left(T_{N V_{1}}^{-}\left(x_{i}\right), T_{N V_{2}}^{-}\left(x_{i}\right)\right), \min \left(T_{N V_{1}}^{+}\left(x_{i}\right), T_{N V_{2}}^{+}\left(x_{i}\right)\right)\right], \\
\hat{I}_{N V}\left(x_{i}\right)=\left[\max \left(I_{N V_{1}}^{-}\left(x_{i}\right), I_{N V_{2}}^{-}\left(x_{i}\right)\right), \max \left(I_{N V_{1}}^{+}\left(x_{i}\right), I_{N V_{2}}^{+}\left(x_{i}\right)\right)\right], \\
\text { and } \hat{F}_{N V}\left(x_{i}\right)=\left[\max \left(F_{N V_{1}}^{-}\left(x_{i}\right), F_{N V_{2}}^{-}\left(x_{i}\right)\right), \max \left(F_{N V_{1}}^{+}\left(x_{i}\right), F_{N V_{2}}^{+}\left(x_{i}\right)\right)\right] .
\end{gathered}
$$

Example 6. We consider the two neutrosophic vague 5-soft sets $\left(\mathrm{NV}_{2}, \mathrm{~K}_{2}\right)$ and $\left(\mathrm{NV}_{3}, \mathrm{~K}_{3}\right)$ defined in Example 2 and 4 respectively. $\left(N V_{2}, K_{2}\right) \widetilde{\cap}_{E}\left(N V_{3}, K_{3}\right)$ can be obtained from Table 8.

Table 8. The $\left(N V_{2}, K_{2}\right) \widetilde{\cap}_{E}\left(N V_{3}, K_{3}\right)$ in Example 6.

\begin{tabular}{ccc}
\hline$\left(N V_{2}, K_{2}\right) \widetilde{\cap}_{E}\left(N V_{3}, K_{3}\right)$ & $e_{1}$ & $e_{2}$ \\
\hline$x_{1}$ & $<3,[0.30,0.90],[0.10,0.70],[0.10,0.70]>$ & $<2,[0.20,0.40],[0.50,0.80],[0.60,0.80]>$ \\
$x_{2}$ & $<3,[0.40,0.80],[0.20,0.50],[0.20,0.60]>$ & $<3,[0.50,0.90],[0.30,0.50],[0.10,0.50]>$ \\
$x_{3}$ & $<2,[0.30,0.40],[0.20,0.40],[0.60,0.70]>$ & $<3,[0.40,0.70],[0.20,0.40],[0.30,0.60]>$ \\
\hline$\left(N V_{2}, K_{2}\right) \widetilde{\cap}_{E}\left(N V_{3}, K_{3}\right)$ & $e_{3}$ & $e_{4}$ \\
\hline$x_{1}$ & $<3,[0.50,0.80],[0.10,0.90],[0.20,0.50]>$ & $<4,[0.80,0.90],[0.50,0.70],[0.10,0.20]>$ \\
$x_{2}$ & $<4,[0.70,0.90],[0.30,0.50],[0.10,0.30]>$ & $<4,[0.70,0.90],[0.30,0.60],[0.10,0.30]>$ \\
$x_{3}$ & $<4,[0.80,0.90],[0.30,0.50],[0.10,0.20]>$ & $<3,[0.60,0.80],[0.20,0.50],[0.20,0.40]>$ \\
\hline
\end{tabular}

Definition 19. Let $U$ be universe of objects, and let $\left(N V_{1}, K_{1}\right)$ and $\left(N V_{2}, K_{2}\right)$ be $N V N S S s, K_{1}=\left(N V_{1}, T, N_{1}\right)$ and $K_{2}=\left(N_{2}, S, N_{2}\right)$ are $N$-soft sets on $U$ at the same $k$-degree risk value. Then their extended union is denoted by $\left(N V_{1}, K_{1}\right) \widetilde{\cup}_{E}\left(N V_{2}, K_{2}\right)$ and it is defined as $\left(\rho_{N V}, K_{1} \cup \mathcal{E} K_{2}\right)$, where $K_{1} \cup \mathcal{E} K_{2}=\left(B, T \cup S, \max \left(N_{1}, N_{2}\right)\right)$, $\forall e_{j} \in T \cup S$ and $x_{i} \in U,<\left(x_{i}, g_{i j}\right), \hat{T}_{N V}\left(x_{i}\right), \hat{I}_{N V}\left(x_{i}\right), \hat{F}_{N V}\left(x_{i}\right)>\in \rho_{N V}\left(e_{j}\right) \Leftrightarrow$

(1) $e_{j} \in T-S$ :

$$
\begin{gathered}
g_{i j}=g_{i j}^{1}, \hat{T}_{N V}\left(x_{i}\right)=\left[T_{N V_{1}}^{-}\left(x_{i}\right), T_{N V_{1}}^{+}\left(x_{i}\right)\right], \\
\hat{I}_{N V}\left(x_{i}\right)=\left[I_{N V_{1}}^{-}\left(x_{i}\right), I_{N V_{1}}^{+}\left(x_{i}\right)\right], \text { and } \hat{F}_{N V}\left(x_{i}\right)=\left[F_{N V_{1}}^{-}\left(x_{i}\right), F_{N V_{1}}^{+}\left(x_{i}\right)\right] ;
\end{gathered}
$$

(2) $e_{j} \in S-T$ :

$$
\begin{gathered}
g_{i j}=g_{i j^{\prime}}^{2} \hat{T}_{N V}\left(x_{i}\right)=\left[T_{N V_{2}}^{-}\left(x_{i}\right), T_{N V_{2}}^{+}\left(x_{i}\right)\right], \\
\hat{I}_{N V}\left(x_{i}\right)=\left[I_{N V_{2}}^{-}\left(x_{i}\right), I_{N V_{2}}^{+}\left(x_{i}\right)\right], \text { and } \hat{F}_{N V}\left(x_{i}\right)=\left[F_{N V_{2}}^{-}\left(x_{i}\right), F_{N V_{2}}^{+}\left(x_{i}\right)\right] ;
\end{gathered}
$$

(3) $e_{j} \in T \cap S$ :

$$
\begin{gathered}
g_{i j}=\max \left(g_{i j}^{1}, g_{i j}^{2}\right), \\
\hat{T}_{N V}\left(x_{i}\right)=\left[\max \left(T_{N V_{1}}^{-}\left(x_{i}\right), T_{N V_{2}}^{-}\left(x_{i}\right)\right), \max \left(T_{N V_{1}}^{+}\left(x_{i}\right), T_{N V_{2}}^{+}\left(x_{i}\right)\right)\right], \\
\hat{I}_{N V}\left(x_{i}\right)=\left[\min \left(I_{N V_{1}}^{-}\left(x_{i}\right), I_{N V_{2}}^{-}\left(x_{i}\right)\right), \min \left(I_{N V_{1}}^{+}\left(x_{i}\right), I_{N V_{2}}^{+}\left(x_{i}\right)\right)\right], \\
\text { and } \hat{F}_{N V}\left(x_{i}\right)=\left[\min \left(F_{N V_{1}}^{-}\left(x_{i}\right), F_{N V_{2}}^{-}\left(x_{i}\right)\right), \min \left(F_{N V_{1}}^{+}\left(x_{i}\right), F_{N V_{2}}^{+}\left(x_{i}\right)\right)\right] .
\end{gathered}
$$

Example 7. We consider the two neutrosophic vague 5-soft sets $\left(\mathrm{NV}_{2}, \mathrm{~K}_{2}\right)$ and $\left(\mathrm{NV}_{3}, \mathrm{~K}_{3}\right)$ defined in Example 2 and 4 respectively. $\left(N V_{2}, K_{2}\right) \widetilde{\cup}_{E}\left(N V_{3}, K_{3}\right)$ can be obtained from Table 9. 
Table 9. The $\left(N V_{2}, K_{2}\right) \widetilde{U}_{E}\left(N V_{3}, K_{3}\right)$ in Example 7.

\begin{tabular}{ccc}
\hline$\left(N V_{2}, K_{2}\right) \widetilde{\cup}_{E}\left(N V_{3}, K_{3}\right)$ & $e_{1}$ & $e_{2}$ \\
\hline$x_{1}$ & $<3,[0.60,0.90],[0.10,0.40],[0.10,0.40]>$ & $<3,[0.40,0.70],[0.20,0.60],[0.30,0.60]>$ \\
$x_{2}$ & $<3,[0.70,0.80],[0.20,0.40],[0.10,0.30]>$ & $<4,[0.70,0.90],[0.20,0.30],[0.10,0.30]>$ \\
$x_{3}$ & $<3,[0.50,0.80],[0.10,0.30],[0.20,0.50]>$ & $<3,[0.60,0.70],[0.20,0.30],[0.30,0.40]>$ \\
\hline$\left(N V_{2}, K_{2}\right) \widetilde{\cup}_{E}\left(N V_{3}, K_{3}\right)$ & $e_{3}$ & $e_{4}$ \\
\hline$x_{1}$ & $<3,[0.50,0.80],[0.10,0.90],[0.20,0.50]>$ & $<4,[0.80,0.90],[0.50,0.70],[0.10,0.20]>$ \\
$x_{2}$ & $<4,[0.70,0.90],[0.30,0.50],[0.10,0.30]>$ & $<4,[0.70,0.90],[0.30,0.60],[0.10,0.30]>$ \\
$x_{3}$ & $<4,[0.80,0.90],[0.30,0.50],[0.10,0.20]>$ & $<3,[0.60,0.80],[0.20,0.50],[0.20,0.40]>$ \\
\hline
\end{tabular}

Proposition 2. Let $\left(N V_{1}, K_{1}\right)$ be a NVNSS, $A_{N V}$ be a neutrosophic absolute vague $N$-soft set, $\varnothing_{N V}$ be a neutrosophic null vague $N$-soft set. Suppose $S$ is the parameter set of $\left(N V_{1}, K_{1}\right), T$ is the parameter set of $A_{N V}$ and $\varnothing_{N V}$ parameter set, then:

(1) $\left(N V_{1}, K_{1}\right) \widetilde{\cup}_{R} \varnothing_{N V}=\left(N V_{1}, K_{1}\right) \Leftrightarrow S \subseteq T$;

(2) $\left(N V_{1}, K_{1}\right) \widetilde{\cap}_{R} \varnothing_{N V}=\varnothing_{N V} \Leftrightarrow T \subseteq S$;

(3) $\left(N V_{1}, K_{1}\right) \widetilde{\cup}_{R} A_{N V}=A_{N V} \Leftrightarrow T \subseteq S$;

(4) $\left(N V_{1}, K_{1}\right) \widetilde{\cap}_{R} A_{N V}=\left(N V_{1}, K_{1}\right) \Leftrightarrow S \subseteq T$.

Proof. (1) Let $U$ be a universe, and $\left(N V_{1}, K_{1}\right)$ be a $N V N S S$, and $\varnothing_{N V}$ (denoted by $\left(N V_{2}, K_{2}\right)$ ) be a neutrosophic null vague $N$-soft set, $K_{1}=\left(N V_{1}, S, N_{1}\right)$ and $K_{2}=\left(N V_{2}, T, N_{2}\right)$ are $N$-soft sets on $U$ at the same $k$-degree risk value. From Definition 13 , we know that $\varnothing_{N V}\left(e_{j}\right)=\left\{<\left(x_{1}, 0\right),[0,0],[1,1]\right.$, $\left.[1,1]>, \cdots,<\left(x_{n}, 0\right),[0,0],[1,1],[1,1]>\right\}$, where $\forall e_{j} \in T$.

From Definition 17, $\left(N V_{1}, K_{1}\right) \widetilde{\cup}_{R} \varnothing_{N V}$ can be defined as $\left(\zeta_{N V}, K_{1} \cup_{\mathcal{R}} K_{2}\right)$, where $K_{1} \cup_{\mathcal{R}} K_{2}=$ $\left(E, S \cap T, \max \left(N_{1}, N_{2}\right)=\max \left(N_{1}, 0\right)=N_{1}, \forall e_{j} \in S \cap T\right.$ and $x_{i} \in U,<\left(x_{i}, g_{i j}\right), \hat{T}_{N V}\left(x_{i}\right), \hat{I}_{N V}\left(x_{i}\right)$, $\hat{F}_{N V}\left(x_{i}\right)>\in \zeta_{N V}\left(e_{j}\right) \Leftrightarrow$

$$
\begin{gathered}
g_{i j}=\max \left(g_{i j}^{1}, g_{i j}^{2}\right)=\max \left(g_{i j}^{1}, 0\right)=g_{i j}^{1}, \\
\hat{T}_{N V}\left(x_{i}\right)=\left[\max \left(T_{N V_{1}}^{-}\left(x_{i}\right), T_{N V_{2}}^{-}\left(x_{i}\right)\right), \max \left(T_{N V_{1}}^{+}\left(x_{i}\right), T_{N V_{2}}^{+}\left(x_{i}\right)\right)\right]=\left[T_{N V_{1}}^{-}\left(x_{i}\right), T_{N V_{1}}^{+}\left(x_{i}\right)\right], \\
\hat{I}_{N V}\left(x_{i}\right)=\left[\min \left(I_{N V_{1}}^{-}\left(x_{i}\right), \min \left(I_{N V_{2}}^{-}\left(x_{i}\right)\right), \min \left(I_{N V_{1}}^{+}\left(x_{i}\right), I_{N V_{2}}^{+}\left(x_{i}\right)\right)\right]=\left[I_{N V_{1}}^{-}\left(x_{i}\right), I_{N V_{1}}^{+}\left(x_{i}\right)\right],\right. \\
\text { and } \hat{F}_{N V}\left(x_{i}\right)=\left[\min \left(F_{N V_{1}}^{-}\left(x_{i}\right), F_{N V_{2}}^{-}\left(x_{i}\right)\right), \min \left(F_{N V_{1}}^{+}\left(x_{i}\right), F_{N V_{2}}^{+}\left(x_{i}\right)\right)\right]=\left[F_{N V_{1}}^{-}\left(x_{i}\right), F_{N V_{1}}^{+}\left(x_{i}\right)\right] . \\
\text { So }\left(N V_{1}, K_{1}\right) \widetilde{\cup}_{R} \varnothing_{N V}=\left(N V_{1}, K_{1}\right) \Leftrightarrow S \cap T=S \Leftrightarrow S \subseteq T .
\end{gathered}
$$

The proof of other propositions is similar by the Definitions 16 and 17 .

Proposition 3. Let $\left(N V_{1}, K_{1}\right)$ be a NVNSS, $A_{N V}$ be a neutrosophic absolute vague $N$-soft set, $\varnothing_{N V}$ be a neutrosophic null vague $N$-soft set. Suppose $S$ is the parameter set of $\left(N V_{1}, K_{1}\right), T$ is the parameter set of $A_{N V}$ and $\varnothing_{N V}$ parameter set, then:

(1) $\left(N V_{1}, K_{1}\right) \widetilde{\cup}_{E} \varnothing_{N V}=\left(N V_{1}, K_{1}\right) \Leftrightarrow T \subseteq S$;

(2) $\left(N V_{1}, K_{1}\right) \widetilde{\cap}_{E} \varnothing_{N V}=\varnothing_{N V} \Leftrightarrow S \subseteq T$;

(3) $\left(N V_{1}, K_{1}\right) \widetilde{\cup}_{E} A_{N V}=A_{N V} \Leftrightarrow S \subseteq T$;

(4) $\left(N V_{1}, K_{1}\right) \widetilde{\cap}_{E} A_{N V}=\left(N V_{1}, K_{1}\right) \Leftrightarrow T \subseteq S$.

Proof. (1) Let $U$ be a universe, and $\left(N V_{1}, K_{1}\right)$ be a NVNSS, and $\varnothing_{N V}$ (denoted by $\left(N V_{2}, K_{2}\right)$ ) be a neutrosophic null vague $N$-soft set, $K_{1}=\left(N V_{1}, S, N_{1}\right)$ and $K_{2}=\left(N V_{2}, T, N_{2}\right)$ are $N$-soft sets on $U$ at the same $k$-degree risk value. From Definition 13 , we know that $\varnothing_{N V}\left(e_{j}\right)=\left\{<\left(x_{1}, 0\right),[0,0],[1,1]\right.$, $\left.[1,1]>, \cdots,<\left(x_{n}, 0\right),[0,0],[1,1],[1,1]>\right\}$, where $\forall e_{j} \in T$.

From Definition 19, $\left(N V_{1}, K_{1}\right) \widetilde{\cup}_{E} \varnothing_{N V}$ can be defined as $\left(\rho_{N V}, K_{1} \cup \mathcal{E} K_{2}\right)$, where $K_{1} \cup_{\mathcal{E}} K_{2}=$ $\left(B, S \cup T, \max \left(N_{1}, N_{2}\right)\right), \forall e_{j} \in S \cup T$ and $x_{i} \in U,<\left(x_{i}, g_{i j}\right), \hat{T}_{N V}\left(x_{i}\right), \hat{I}_{N V}\left(x_{i}\right), \hat{F}_{N V}\left(x_{i}\right)>\in \rho_{N V}\left(e_{j}\right) \Leftrightarrow$ 
(i) $e_{j} \in S-T$ :

$$
\begin{gathered}
g_{i j}=g_{i j}^{1}, \hat{T}_{N V}\left(x_{i}\right)=\left[T_{N V_{1}}^{-}\left(x_{i}\right), T_{N V_{1}}^{+}\left(x_{i}\right)\right], \\
\hat{I}_{N V}\left(x_{i}\right)=\left[I_{N V_{1}}^{-}\left(x_{i}\right), I_{N V_{1}}^{+}\left(x_{i}\right)\right], \text { and } \hat{F}_{N V}\left(x_{i}\right)=\left[F_{N V_{1}}^{-}\left(x_{i}\right), F_{N V_{1}}^{+}\left(x_{i}\right)\right] ;
\end{gathered}
$$

(ii) $e_{j} \in T-S$ :

$$
\begin{gathered}
g_{i j}=g_{i j}^{2}=0, \hat{T}_{N V}\left(x_{i}\right)=\left[T_{N V_{2}}^{-}\left(x_{i}\right), T_{N V_{2}}^{+}\left(x_{i}\right)\right]=[0,0], \\
\hat{I}_{N V}\left(x_{i}\right)=\left[I_{N V_{2}}^{-}\left(x_{i}\right), I_{N V_{2}}^{+}\left(x_{i}\right)\right]=[1,1], \text { and } \hat{F}_{N V}\left(x_{i}\right)=\left[F_{N V_{2}}^{-}\left(x_{i}\right), F_{N V_{2}}^{+}\left(x_{i}\right)\right]=[1,1] ;
\end{gathered}
$$

(iii) $e_{j} \in T \cap S$ :

$$
\begin{gathered}
g_{i j}=\max \left(g_{i j}^{1} g_{i j}^{2}\right)=g_{i j^{\prime}}^{1} \\
\hat{T}_{N V}\left(x_{i}\right)=\left[\max \left(T_{N V_{1}}^{-}\left(x_{i}\right), T_{N V_{2}}^{-}\left(x_{i}\right)\right), \max \left(T_{N V_{1}}^{+}\left(x_{i}\right), T_{N V_{2}}^{+}\left(x_{i}\right)\right)\right]=\left[T_{N V_{1}}^{-}\left(x_{i}\right), T_{N V_{1}}^{+}\left(x_{i}\right)\right], \\
\hat{I}_{N V}\left(x_{i}\right)=\left[\min \left(I_{N V_{1}}^{-}\left(x_{i}\right), I_{N V_{2}}^{-}\left(x_{i}\right)\right), \min \left(I_{N V_{1}}^{+}\left(x_{i}\right), I_{N V_{2}}^{+}\left(x_{i}\right)\right)\right]=\left[I_{N V_{1}}^{-}\left(x_{i}\right), I_{N V_{1}}^{+}\left(x_{i}\right)\right], \\
\text { and } \hat{F}_{N V}\left(x_{i}\right)=\left[\min \left(F_{N V_{1}}^{-}\left(x_{i}\right), F_{N V_{2}}^{-}\left(x_{i}\right)\right), \min \left(F_{N V_{1}}^{+}\left(x_{i}\right), F_{N V_{2}}^{+}\left(x_{i}\right)\right)\right]=\left[F_{N V_{1}}^{-}\left(x_{i}\right), F_{N V_{1}}^{+}\left(x_{i}\right)\right] .
\end{gathered}
$$

So $\left(N V_{1}, K_{1}\right) \widetilde{\cup}_{E} \varnothing_{N V}=\left(N V_{1}, K_{1}\right) \Leftrightarrow T \cap S=T \Leftrightarrow T \subseteq S$.

The proof of other propositions is similar by the Definitions 18 and 19.

Proposition 4. Let $\left(N V_{1}, K_{1}\right),\left(N V_{2}, K_{2}\right)$ and $\left(N V_{3}, K_{3}\right)$ be three $N V N S S s$, then:

(1) $\left(N V_{1}, K_{1}\right) \widetilde{\cup}_{E}\left(N V_{2}, K_{2}\right)=\left(N V_{2}, K_{2}\right) \widetilde{\cup}_{E}\left(N V_{1}, K_{1}\right)$;

(2) $\left(N V_{1}, K_{1}\right) \widetilde{\cap}_{E}\left(N V_{2}, K_{2}\right)=\left(N V_{2}, K_{2}\right) \widetilde{\cap}_{E}\left(N V_{1}, K_{1}\right)$;

(3) $\left(N V_{1}, K_{1}\right) \widetilde{\cup}_{R}\left(N V_{2}, K_{2}\right)=\left(N V_{2}, K_{2}\right) \widetilde{\cup}_{R}\left(N V_{1}, K_{1}\right)$;

(4) $\left(N V_{1}, K_{1}\right) \widetilde{\cap}_{R}\left(N V_{2}, K_{2}\right)=\left(N V_{2}, K_{2}\right) \widetilde{\cap}_{R}\left(N V_{1}, K_{1}\right)$;

(5) $\quad\left(N V_{1}, K_{1}\right) \widetilde{\cup}_{R}\left(\left(N V_{2}, K_{2}\right) \widetilde{\cup}_{R}\left(N V_{3}, K_{3}\right)\right)=\left(\left(N V_{1}, K_{1}\right) \widetilde{\cup}_{R}\left(N V_{2}, K_{2}\right)\right) \widetilde{\cup}_{R}\left(N V_{3}, K_{3}\right)$;

(6) $\left(N V_{1}, K_{1}\right) \widetilde{\cap}_{R}\left(\left(N V_{2}, K_{2}\right) \widetilde{\cap}_{R}\left(N V_{3}, K_{3}\right)\right)=\left(\left(N V_{1}, K_{1}\right) \widetilde{\cap}_{R}\left(N V_{2}, K_{2}\right)\right) \widetilde{\cap}_{R}\left(N V_{3}, K_{3}\right)$;

(7) $\left(N V_{1}, K_{1}\right) \widetilde{\cup}_{R}\left(\left(N V_{2}, K_{2}\right) \widetilde{\cap}_{R}\left(N V_{3}, K_{3}\right)\right)=\left(\left(N V_{1}, K_{1}\right) \widetilde{\cup}_{R}\left(N V_{2}, K_{2}\right)\right) \widetilde{\cap}_{R}\left(\left(N V_{1}, K_{1}\right) \widetilde{\cup}_{R}\left(N V_{3}, K_{3}\right)\right)$;

(8) $\left(N V_{1}, K_{1}\right) \widetilde{\cap}_{R}\left(\left(N V_{2}, K_{2}\right) \widetilde{\cup}_{R}\left(N V_{3}, K_{3}\right)\right)=\left(\left(N V_{1}, K_{1}\right) \widetilde{\cap}_{R}\left(N V_{2}, K_{2}\right)\right) \widetilde{\cup}_{R}\left(\left(N V_{1}, K_{1}\right) \widetilde{\cap}_{R}\left(N V_{3}, K_{3}\right)\right)$.

Proof. (5) Let $U$ be a universe, and $\left(N V_{l}, K_{l}\right)$ are $N V N S S s, l=1,2, \ldots, 7$. Supposed that $\left(N V_{2}, K_{2}\right) \widetilde{\cup}_{R}\left(N V_{3}, K_{3}\right)=\left(N V_{4}, K_{4}\right), K_{2}=\left(N V_{2}, S_{2}, N_{2}\right), K_{3}=\left(N V_{3}, S_{3}, N_{3}\right)$ are $N$-soft sets on $U$ at the same $k$-degree risk value.

From Definition 17, $\left(N V_{4}, K_{4}\right)$ can be defined as $\left(\zeta_{N V_{23}}, K_{2} \cup_{\mathcal{R}} K_{3}\right)$, where $K_{4}=\left(E, S_{2} \cap\right.$ $\left.S_{3}, \max \left(N_{2}, N_{3}\right)\right), \forall e_{j} \in S_{2} \cap S_{3}$ and $x_{i} \in U,<\left(x_{i}, g_{i j}\right), \hat{T}_{N V}\left(x_{i}\right), \hat{I}_{N V}\left(x_{i}\right), \hat{F}_{N V}\left(x_{i}\right)>\in \zeta_{N V_{23}}\left(e_{j}\right) \Leftrightarrow$

$$
\begin{gathered}
g_{i j}=\max \left(g_{i j}^{2}, g_{i j}^{3}\right), \\
\hat{T}_{N V}\left(x_{i}\right)=\left[\max \left(T_{N V_{2}}^{-}\left(x_{i}\right), T_{N V_{3}}^{-}\left(x_{i}\right)\right), \max \left(T_{N V_{2}}^{+}\left(x_{i}\right), T_{N V_{3}}^{+}\left(x_{i}\right)\right)\right], \\
\hat{I}_{N V}\left(x_{i}\right)=\left[\min \left(I_{N V_{2}}^{-}\left(x_{i}\right), \min \left(I_{N V_{3}}^{-}\left(x_{i}\right)\right), \min \left(I_{N V_{2}}^{+}\left(x_{i}\right), I_{N V_{3}}^{+}\left(x_{i}\right)\right)\right],\right. \\
\text { and } \hat{F}_{N V}\left(x_{i}\right)=\left[\min \left(F_{N V_{2}}^{-}\left(x_{i}\right), F_{N V_{3}}^{-}\left(x_{i}\right)\right), \min \left(F_{N V_{2}}^{+}\left(x_{i}\right), F_{N V_{3}}^{+}\left(x_{i}\right)\right)\right] .
\end{gathered}
$$

Supposed that $\left(N V_{1}, K_{1}\right) \widetilde{\cup}_{R}\left(N V_{2}, K_{2}\right)=\left(N V_{5}, K_{5}\right), K_{1}=\left(N V_{1}, S_{1}, N_{1}\right), K_{2}=\left(N V_{2}, S_{2}, N_{2}\right)$ are $\mathrm{N}$-soft sets on $U$ at the same $k$-degree risk value. And by the same way, we can get $\left(N V_{5}, K_{5}\right)$ can 
be defined as $\left(\zeta_{N V_{12},}, K_{1} \cup_{\mathcal{R}} K_{2}\right)$, where $K_{5}=\left(E, S_{1} \cap S_{2}, \max \left(N_{1}, N_{2}\right)\right), \forall e_{j} \in S_{1} \cap S_{2}$ and $x_{i} \in U$, $<\left(x_{i}, g_{i j}\right), \hat{T}_{N V}\left(x_{i}\right), \hat{I}_{N V}\left(x_{i}\right), \hat{F}_{N V}\left(x_{i}\right)>\in \zeta_{N V_{12}}\left(e_{j}\right) \Leftrightarrow$

$$
\begin{gathered}
g_{i j}=\max \left(g_{i j}^{1}, g_{i j}^{2}\right), \\
\hat{T}_{N V}\left(x_{i}\right)=\left[\max \left(T_{N V_{1}}^{-}\left(x_{i}\right), T_{N V_{2}}^{-}\left(x_{i}\right)\right), \max \left(T_{N V_{1}}^{+}\left(x_{i}\right), T_{N V_{2}}^{+}\left(x_{i}\right)\right)\right], \\
\hat{I}_{N V}\left(x_{i}\right)=\left[\min \left(I_{N V_{1}}^{-}\left(x_{i}\right), \min \left(I_{N V_{2}}^{-}\left(x_{i}\right)\right), \min \left(I_{N V_{1}}^{+}\left(x_{i}\right), I_{N V_{2}}^{+}\left(x_{i}\right)\right)\right],\right. \\
\text { and } \hat{F}_{N V}\left(x_{i}\right)=\left[\min \left(F_{N V_{1}}^{-}\left(x_{i}\right), F_{N V_{2}}^{-}\left(x_{i}\right)\right), \min \left(F_{N V_{1}}^{+}\left(x_{i}\right), F_{N V_{2}}^{+}\left(x_{i}\right)\right)\right] .
\end{gathered}
$$

Supposed that $\left(N V_{1}, K_{1}\right) \widetilde{\cup}_{R}\left(N V_{4}, K_{4}\right)=\left(N V_{6}, K_{6}\right), K_{1}=\left(N V_{1}, S_{1}, N_{1}\right), K_{4}=\left(N V_{4}, S_{4}, N_{4}\right)$ are $N$-soft sets on $U$ at the same $k$-degree risk value. And by the same way, we can get $\left(N V_{6}, K_{6}\right)$ can be defined as $\left(\zeta_{N V_{14}}, K_{1} \cup_{\mathcal{R}} K_{4}\right)$, where $K_{1} \cup_{\mathcal{R}} K_{4}=\left(E, S_{1} \cap S_{4}=S_{1} \cap\left(S_{2} \cap S_{3}\right), \max \left(N_{1}, \max \left(N_{2}, N_{3}\right)\right)\right.$. So $K_{6}=\left(E, S_{1} \cap S_{2} \cap S_{3}, \max \left(N_{1}, N_{2}, N_{3}\right), \forall e_{j} \in S_{1} \cap S_{2} \cap S_{3}\right.$ and $x_{i} \in U,<\left(x_{i}, g_{i j}\right), \hat{T}_{N V}\left(x_{i}\right), \hat{I}_{N V}\left(x_{i}\right)$, $\hat{F}_{N V}\left(x_{i}\right)>\in \zeta_{N V_{14}}\left(e_{j}\right) \Leftrightarrow$

$$
\begin{gathered}
g_{i j}=\max \left(g_{i j}^{1}, \max \left(g_{i j}^{2}, g_{i j}^{3}\right)\right)=\max \left(g_{i j}^{1}, g_{i j}^{2}, g_{i j}^{3}\right), \\
\begin{aligned}
\hat{T}_{N V}\left(x_{i}\right)= & {\left[\max \left(T_{N V_{1}}^{-}\left(x_{i}\right), \max \left(T_{N V_{2}}^{-}\left(x_{i}\right), T_{N V_{3}}^{-}\left(x_{i}\right)\right)\right), \max \left(T_{N V_{1}}^{+}\left(x_{i}\right), \max \left(T_{N V_{2}}^{+}\left(x_{i}\right), T_{N V_{3}}^{+}\left(x_{i}\right)\right)\right)\right] } \\
= & {\left[\max \left(T_{N V_{1}}^{-}\left(x_{i}\right), T_{N V_{2}}^{-}\left(x_{i}\right), T_{N V_{3}}^{-}\left(x_{i}\right)\right), \max \left(T_{N V_{1}}^{+}\left(x_{i}\right), T_{N V_{2}}^{+}\left(x_{i}\right), T_{N V_{3}}^{+}\left(x_{i}\right)\right)\right], } \\
\hat{I}_{N V}\left(x_{i}\right)= & {\left[\min \left(I_{N V_{1}}^{-}\left(x_{i}\right), \min \left(I_{N V_{2}}^{-}\left(x_{i}\right), I_{N V_{3}}^{-}\left(x_{i}\right)\right)\right), \min \left(I_{N V_{1}}^{+}\left(x_{i}\right), \min \left(I_{N V_{2}}^{+}\left(x_{i}\right), I_{N V_{3}}^{+}\left(x_{i}\right)\right)\right)\right] } \\
& =\left[\min \left(I_{N V_{1}}^{-}\left(x_{i}\right), I_{N V_{2}}^{-}\left(x_{i}\right), I_{N V_{3}}^{-}\left(x_{i}\right)\right), \min \left(I_{N V_{1}}^{+}\left(x_{i}\right), I_{N V_{2}}^{+}\left(x_{i}\right), I_{N V_{3}}^{+}\left(x_{i}\right)\right)\right], \\
\text { and } \hat{F}_{N V}\left(x_{i}\right) & =\left[\min \left(F_{N V_{1}}^{-}\left(x_{i}\right), \min \left(F_{N V_{2}}^{-}\left(x_{i}\right), F_{N V_{3}}^{-}\left(x_{i}\right)\right)\right), \min \left(F_{N V_{1}}^{+}\left(x_{i}\right), \min \left(F_{N V_{2}}^{+}\left(x_{i}\right), F_{N V_{3}}^{+}\left(x_{i}\right)\right)\right)\right] \\
& =\left[\min \left(F_{N V_{1}}^{-}\left(x_{i}\right), F_{N V_{2}}^{-}\left(x_{i}\right), F_{N V_{3}}^{-}\left(x_{i}\right)\right), \min \left(F_{N V_{1}}^{+}\left(x_{i}\right), F_{N V_{2}}^{+}\left(x_{i}\right), F_{N V_{3}}^{+}\left(x_{i}\right)\right)\right] .
\end{aligned}
\end{gathered}
$$

Supposed that $\left(N V_{5}, K_{5}\right) \widetilde{\cup}_{R}\left(N V_{3}, K_{3}\right)=\left(N V_{7}, K_{7}\right), K_{5}=\left(N V_{5}, S_{5}, N_{5}\right), K_{3}=\left(N V_{3}, S_{3}, N_{3}\right)$ are $N$-soft sets on $U$ at the same $k$-degree risk value. And by the same way, we can get $\left(N V_{7}, K_{7}\right)$ can be defined as $\left(\zeta_{N V_{53}}, K_{5} \cup_{\mathcal{R}} K_{3}\right)$, where $K_{5} \cup_{\mathcal{R}} K_{3}=\left(E, S_{5} \cap S_{3}=\left(S_{1} \cap S_{2}\right) \cap S_{3}, \max \left(\max \left(N_{1}, N_{2}\right), N_{3}\right)\right.$. So $K_{7}=\left(E, S_{1} \cap S_{2} \cap S_{3}, \max \left(N_{1}, N_{2}, N_{3}\right), \forall e_{j} \in S_{1} \cap S_{2} \cap S_{3}\right.$ and $x_{i} \in U,<\left(x_{i}, g_{i j}\right), \hat{T}_{N V}\left(x_{i}\right), \hat{I}_{N V}\left(x_{i}\right)$, $\hat{F}_{N V}\left(x_{i}\right)>\in \zeta_{N V_{53}}\left(e_{j}\right) \Leftrightarrow$

$$
\begin{gathered}
g_{i j}=\max \left(\max \left(g_{i j}^{1}, g_{i j}^{2}\right), g_{i j}^{3}\right)=\max \left(g_{i j}^{1}, g_{i j}^{2}, g_{i j}^{3}\right), \\
\begin{aligned}
& \hat{T}_{N V}\left(x_{i}\right)= {\left[\max \left(\max \left(T_{N V_{1}}^{-}\left(x_{i}\right), T_{N V_{2}}^{-}\left(x_{i}\right)\right), T_{N V_{3}}^{-}\left(x_{i}\right)\right), \max \left(\max \left(T_{N V_{1}}^{+}\left(x_{i}\right), T_{N V_{2}}^{+}\left(x_{i}\right)\right), T_{N V_{3}}^{+}\left(x_{i}\right)\right)\right] } \\
&= {\left[\max \left(T_{N V_{1}}^{-}\left(x_{i}\right), T_{N V_{2}}^{-}\left(x_{i}\right), T_{N V_{3}}^{-}\left(x_{i}\right)\right), \max \left(T_{N V_{1}}^{+}\left(x_{i}\right), T_{N V_{2}}^{+}\left(x_{i}\right), T_{N V_{3}}^{+}\left(x_{i}\right)\right)\right], } \\
& \hat{I}_{N V}\left(x_{i}\right)=\left[\min \left(\min \left(I_{N V_{1}}^{-}\left(x_{i}\right), I_{N V_{2}}^{-}\left(x_{i}\right)\right), I_{N V_{3}}^{-}\left(x_{i}\right)\right), \min \left(\min \left(I_{N V_{1}}^{+}\left(x_{i}\right), I_{N V_{2}}^{+}\left(x_{i}\right)\right), I_{N V_{3}}^{+}\left(x_{i}\right)\right)\right] \\
&=\left[\min \left(I_{N V_{1}}^{-}\left(x_{i}\right), I_{N V_{2}}^{-}\left(x_{i}\right), I_{N V_{3}}^{-}\left(x_{i}\right)\right), \min \left(I_{N V_{1}}^{+}\left(x_{i}\right), I_{N V_{2}}^{+}\left(x_{i}\right), I_{N V_{3}}^{+}\left(x_{i}\right)\right)\right], \\
& \text { and } \hat{F}_{N V}\left(x_{i}\right)=\left[\min \left(\min \left(F_{N V_{1}}^{-}\left(x_{i}\right), F_{N V_{2}}^{-}\left(x_{i}\right)\right), F_{N V_{3}}^{-}\left(x_{i}\right)\right), \min \left(\min \left(F_{N V_{1}}^{+}\left(x_{i}\right), F_{N V_{2}}^{+}\left(x_{i}\right)\right), F_{N V_{3}}^{+}\left(x_{i}\right)\right)\right] \\
&=\left[\min \left(F_{N V_{1}}^{-}\left(x_{i}\right), F_{N V_{2}}^{-}\left(x_{i}\right), F_{N V_{3}}^{-}\left(x_{i}\right)\right), \min \left(F_{N V_{1}}^{+}\left(x_{i}\right), F_{N V_{2}}^{+}\left(x_{i}\right), F_{N V_{3}}^{+}\left(x_{i}\right)\right)\right] .
\end{aligned} \\
\text { So }\left(N V_{6}, K_{6}\right)=\left(N V_{7}, K_{7}\right) \Leftrightarrow\left(N V_{1}, K_{1}\right) \widetilde{\cup}_{R}\left(\left(N V_{2}, K_{2}\right) \widetilde{\cup}_{R}\left(N V_{3}, K_{3}\right)\right)=\left(\left(N V_{1}, K_{1}\right) \widetilde{\cup}_{R}\left(N V_{2}, K_{2}\right)\right) \widetilde{\cup}_{R}
\end{gathered}
$$
$\left(N V_{3}, K_{3}\right)$.

The proof of other propositions is similar by the Definitions $16-19$.

\section{Multi-Attribute Decision Making Method Based on NVNSSs}

\subsection{Priority Relation Ranking Method Based on NVNSSs}

Practice has proved that people's understanding of things often comes from the comparison of things. And each comparison can get a vague understanding. The fuzzy cognition is quantified and a fuzzy priority relation is established. Then research objects are sorted out in an order of advantages and disadvantages. This is the fuzzy priority relationship ranking decision method. Based on it, priority relation ranking method based on NVNSSs is proposed for the first time.

Let $E=\left\{e_{1}, \cdots, e_{j}, \cdots, e_{m}\right\}$ is a set of parameters considered on $U=\left\{x_{1}, \cdots, x_{i}, \cdots, x_{n}\right\}$. The decision maker has the power to assign weights to each parameter and suppose that the weights 
satisfy the condition: $\sum_{j=1}^{m} \omega_{j}=1, \omega_{j} \in[0,1]$. For the convenience of writing, $T_{N V}^{-}(x)$ is abbreviated as $T^{-}$, and others are similar. The following are specific decision steps:

Step 1. Through the actual data in a problem, we can get a NVNSS denoted by $(N V, K)$ in Table 10. At this moment, the $k$-degree risk value is 0 .

Table 10. The neutrosophic vague $N$-soft sets $(N V, K)$.

\begin{tabular}{cccc}
\hline$(N \boldsymbol{V}, \boldsymbol{K})$ & $\boldsymbol{e}_{\mathbf{1}}$ & $\cdots$ & $\boldsymbol{e}_{\boldsymbol{m}}$ \\
\hline$x_{1}$ & $<g_{11},\left[T^{-}, T^{+}\right],\left[I^{-}, I^{+}\right],\left[F^{-}, F^{+}\right]>$ & $\cdots$ & $<g_{1 m},\left[T^{-}, T^{+}\right],\left[I^{-}, I^{+}\right],\left[F^{-}, F^{+}\right]>$ \\
$x_{2}$ & $<g_{21},\left[T^{-}, T^{+}\right],\left[I^{-}, I^{+}\right],\left[F^{-}, F^{+}\right]>$ & $\cdots$ & $<g_{2 m},\left[T^{-}, T^{+}\right],\left[I^{-}, I^{+}\right],\left[F^{-}, F^{+}\right]>$ \\
$\vdots$ & $\vdots$ & $\vdots$ & $\vdots$ \\
$x_{n}$ & $<g_{n 1},\left[T^{-}, T^{+}\right],\left[I^{-}, I^{+}\right],\left[F^{-}, F^{+}\right]>$ & $\cdots$ & $<g_{n m},\left[T^{-}, T^{+}\right],\left[I^{-}, I^{+}\right],\left[F^{-}, F^{+}\right]>$ \\
\hline
\end{tabular}

Step 2. The first screening was carried out with the grade of 0-degree risk value. Grades representation of the $(N V, K)$ denoted by Table 11.

Table 11. Grades of the $(N V, K)$.

\begin{tabular}{ccccc}
\hline$(\boldsymbol{N} \boldsymbol{V}, \boldsymbol{K})$ & $\boldsymbol{e}_{\mathbf{1}}$ & $\cdots$ & $\boldsymbol{e}_{\boldsymbol{m}}$ & $\boldsymbol{g}_{\text {imin }}$ \\
\hline$x_{1}$ & $g_{11}$ & $\cdots$ & $g_{1 m}$ & $g_{1 \min }$ \\
$x_{2}$ & $g_{21}$ & $\cdots$ & $g_{2 m}$ & $g_{2 \min }$ \\
$\vdots$ & $\vdots$ & $\vdots$ & $\vdots$ & $\vdots$ \\
$x_{n}$ & $g_{n 1}$ & $\cdots$ & $g_{n m}$ & $g_{n \min }$ \\
\hline
\end{tabular}

First, we find the minimum grade $g_{i m i n}$ of the object $x_{i}$ under different attributes. And $G_{i m i n}=$ $\left\{g_{1 \min }, \cdots, g_{\text {imin }}, \cdots, g_{n \text { min }}\right\}$ can be obtained. Then, according to the actual situations and needs, one of the grades in $G_{i \min }$ denoted by $g_{i m i n}$ is selected as the grade threshold for the first screening. Finally, if one of its grades based on different attributes lower than the grade threshold, the research object will be eliminated. Suppose $x_{1}, x_{2}, x_{3}$ can pass the first screening, Table 12 can be obtained easily.

Table 12. The research objects after passing the first screening.

\begin{tabular}{cccc}
\hline$(\boldsymbol{N} \boldsymbol{V}, \boldsymbol{K})$ & $\boldsymbol{e}_{\mathbf{1}}$ & $\cdots$ & $\boldsymbol{e}_{\boldsymbol{m}}$ \\
\hline$x_{1}$ & $<g_{11},\left[T^{-}, T^{+}\right],\left[I^{-}, I^{+}\right],\left[F^{-}, F^{+}\right]>$ & $\cdots$ & $<g_{1 m},\left[T^{-}, T^{+}\right],\left[I^{-}, I^{+}\right],\left[F^{-}, F^{+}\right]>$ \\
$x_{2}$ & $<g_{21},\left[T^{-}, T^{+}\right],\left[I^{-}, I^{+}\right],\left[F^{-}, F^{+}\right]>$ & $\cdots$ & $<g_{2 m},\left[T^{-}, T^{+}\right],\left[I^{-}, I^{+}\right],\left[F^{-}, F^{+}\right]>$ \\
$x_{3}$ & $<g_{31},\left[T^{-}, T^{+}\right],\left[I^{-}, I^{+}\right],\left[F^{-}, F^{+}\right]>$ & $\cdots$ & $<g_{3 m},\left[T^{-}, T^{+}\right],\left[I^{-}, I^{+}\right],\left[F^{-}, F^{+}\right]>$ \\
\hline
\end{tabular}

Step 3. The decision maker determine the $k$-degree risk value based on the question type and his own risk preference. Supposed $k=0.5$, so a new neutrosophic vague $N$-soft set $(N V, K)^{\prime}$ based on 0.5 -degree risk value and $(N V, K)$. Table 13 represents $(N V, K)^{\prime}$.

Table 13. The new neutrosophic vague $N$-soft sets $(N V, K)^{\prime}$.

\begin{tabular}{cccc}
\hline$(\boldsymbol{N} \boldsymbol{V}, \boldsymbol{K})^{\prime}$ & $\boldsymbol{e}_{\mathbf{1}}$ & $\cdots$ & $\boldsymbol{e}_{\boldsymbol{m}}$ \\
\hline$x_{1}$ & $<g_{11}^{\prime},\left[T^{-}, T^{+}\right],\left[I^{-}, I^{+}\right],\left[F^{-}, F^{+}\right]>$ & $\cdots$ & $<g_{1 m^{\prime}}^{\prime}\left[T^{-}, T^{+}\right],\left[I^{-}, I^{+}\right],\left[F^{-}, F^{+}\right]>$ \\
$x_{2}$ & $<g_{21}^{\prime},\left[T^{-}, T^{+}\right],\left[I^{-}, I^{+}\right],\left[F^{-}, F^{+}\right]>$ & $\cdots$ & $<g_{2 m}^{\prime},\left[T^{-}, T^{+}\right],\left[I^{-}, I^{+}\right],\left[F^{-}, F^{+}\right]>$ \\
$x_{3}$ & $<g_{31}^{\prime},\left[T^{-}, T^{+}\right],\left[I^{-}, I^{+}\right],\left[F^{-}, F^{+}\right]>$ & $\cdots$ & $<g_{3 m^{\prime}}^{\prime}\left[T^{-}, T^{+}\right],\left[I^{-}, I^{+}\right],\left[F^{-}, F^{+}\right]>$ \\
\hline
\end{tabular}

Step 4. Compute the score function $S_{i j}^{\prime}$ based on $(N V, K)^{\prime}$ denoted by Table 14.

$$
S_{i j}^{\prime}=T_{i j}^{\prime}-I_{i j}^{\prime}-F_{i j}^{\prime}
$$


where $T_{i j}^{\prime}=T_{i j}^{-}+\hat{k}\left(T_{i j}^{+}-T_{i j}^{-}\right), I_{i j}^{\prime}=I_{i j}^{-}+\hat{k}\left(I_{i j}^{+}-I_{i j}^{-}\right), F_{i j}^{\prime}=F_{i j}^{+}-\hat{k}\left(F_{i j}^{+}-F_{i j}^{-}\right), i=1,2, \cdots, n ; j=$ $1,2, \cdots, m$.

Table 14. The score function $S_{i j}^{\prime}$ based on $(N V, K)^{\prime}$.

\begin{tabular}{cccc}
\hline$(\boldsymbol{N} \boldsymbol{V}, \boldsymbol{K})^{\prime}$ & $\boldsymbol{e}_{\mathbf{1}}$ & $\cdots$ & $\boldsymbol{e}_{m}$ \\
\hline$x_{1}$ & $<g_{11}^{\prime}, T_{11}^{\prime}, I_{11}^{\prime}, F_{11}^{\prime}, S_{11}^{\prime}>$ & $\ldots$ & $<g_{1 m}^{\prime}, T_{1 m}^{\prime}, I_{1 m}^{\prime}, F_{1 m}^{\prime}, S_{1 m}^{\prime}>$ \\
$x_{2}$ & $<g_{21}^{\prime}, T_{21}^{\prime}, I_{21}^{\prime}, F_{21}^{\prime}, S_{21}^{\prime}>$ & $\ldots$ & $<g_{2 m}^{\prime}, T_{2 m}^{\prime}, I_{2 m}^{\prime}, F_{2 m}^{\prime}, S_{2 m}^{\prime}>$ \\
$x_{3}$ & $<g_{31}^{\prime}, T_{31}^{\prime}, I_{31}^{\prime}, F_{31}^{\prime}, S_{31}^{\prime}>$ & $\cdots$ & $<g_{3 m}^{\prime}, T_{3 m}^{\prime}, I_{3 m}^{\prime}, F_{3 m}^{\prime}, S_{3 m}^{\prime}>$ \\
\hline
\end{tabular}

Step 5. Compute the comparison table for $\Delta g_{i i^{*}}^{\prime}$ and $\Delta S_{i i^{*}}^{\prime}$ based on $(N V, K)^{\prime}$ under all the attributes $E$, where

$$
\begin{aligned}
& \Delta g_{i i^{*}}^{\prime}=g_{i j}^{\prime}-g_{i^{*} j^{\prime}}^{\prime} \\
& \Delta S_{i i^{*}}^{\prime}=S_{i j}^{\prime}-S_{i^{*} j}^{\prime} .
\end{aligned}
$$

For example, $\Delta g_{21}^{\prime}=g_{2 j}^{\prime}-g_{1 j}^{\prime}, \Delta S_{21}^{\prime}=S_{2 j}^{\prime}-S_{1 j}^{\prime}, \Delta g_{i i}^{\prime}=0, \Delta S_{i i}^{\prime}=0$ under the same attribute $e_{j}$, and the others are similar. So Table 15 can be given. $\Delta_{\text {imin }}=\left\langle\Delta g_{i}^{\prime}, \Delta S_{i}^{\prime}\right\rangle, i^{*}, i=1,2,3$.

Table 15. Comparison table for $g_{i i^{*}}^{\prime}$ and $S_{i i^{*}}^{\prime}$ based on $(N V, K)^{\prime}$.

\begin{tabular}{ccccc}
\hline For $\boldsymbol{e}_{j}$ & $\boldsymbol{x}_{\mathbf{1}}$ & $\boldsymbol{x}_{\mathbf{2}}$ & $\boldsymbol{x}_{\mathbf{3}}$ & $\boldsymbol{\Delta}_{\text {imin }}$ \\
\hline$x_{1}$ & $<\Delta g_{11}^{\prime}, \Delta S_{11}^{\prime}>$ & $<\Delta g_{12}^{\prime}, \Delta S_{12}^{\prime}>$ & $<\Delta g_{13}^{\prime}, \Delta S_{13}^{\prime}>$ & $<\Delta g_{1}^{\prime}, \Delta S_{1}^{\prime}>$ \\
$x_{2}$ & $<\Delta g_{21}^{\prime}, \Delta S_{21}^{\prime}>$ & $<\Delta g_{22}^{\prime}, \Delta S_{22}^{\prime}>$ & $<\Delta g_{23}^{\prime}, \Delta S_{23}^{\prime}>$ & $<\Delta g_{2}^{\prime}, \Delta S_{2}^{\prime}>$ \\
$x_{3}$ & $<\Delta g_{31}^{\prime}, \Delta S_{31}^{\prime 1}>$ & $<\Delta g_{32}^{\prime}, \Delta S_{32}^{\prime}>$ & $<\Delta g_{33}^{\prime}, \Delta S_{33}^{\prime}>$ & $<\Delta g_{3}^{\prime}, \Delta S_{3}^{\prime}>$ \\
\hline
\end{tabular}

Step 6. Use priority relation method to complete the ranking of the research objects. If $\Delta_{3}=\max \left\{\Delta_{\text {imin }}\right\}, i=1,2,3$. We will remove $x_{3}$ and consider $x_{3}$ is the first superior object under the same attribute $e_{j}$.

At this point, we should delete the row and column of $x_{3}$ in Table 15 and seek the second priority object. If this is not done, the attribute value of the first priority object $x_{3}$ will have an impact on the subsequent rankings so that we can't produce the correct rankings.

If we go on in turn, we can get the orders of all the research objects under the same attribute $e_{j}$.

Step 7. Compute the rankings of the research objects under all attributes. Suppose $E=\left\{e_{1}, e_{2}, e_{3}\right\}$, we can get Table 16 . The decision maker assigns weights to each attribute and $\sum_{j=1}^{m} \omega_{j}=1, \omega_{j} \in[0,1]$.

$$
A_{i}=a_{i 1} \cdot \omega_{1}+a_{i 2} \cdot \omega_{2}+a_{i 3} \cdot \omega_{3}
$$

where $a_{i 1}$ represents the ranking of the research object $x_{i}$ under the attribute $e_{1}$, and others are similar. If $A_{i}=\min \left\{A_{1}, A_{2}, A_{3}\right\}, i=1,2,3$, then $x_{i}$ is the first superior object under the $E$. And if $A_{1}>A_{2}>A_{3}$, we consider that $x_{3}$ is the first superior object, $x_{2}$ is the second superior object and $x_{1}$ is the third superior object under the $E$.

Table 16. The ranking of the objects under all attributes.

\begin{tabular}{ccccc}
\hline & $\boldsymbol{e}_{\mathbf{1}} \cdot \boldsymbol{\omega}_{\mathbf{1}}$ & $\boldsymbol{e}_{\mathbf{2}} \cdot \boldsymbol{\omega}_{\mathbf{2}}$ & $\boldsymbol{e}_{\mathbf{3}} \cdot \boldsymbol{\omega}_{\mathbf{3}}$ & $A_{\boldsymbol{i}}$ \\
\hline$x_{1}$ & $a_{11}$ & $a_{12}$ & $a_{13}$ & $A_{1}$ \\
$x_{2}$ & $a_{21}$ & $a_{22}$ & $a_{23}$ & $A_{2}$ \\
$x_{3}$ & $a_{31}$ & $a_{32}$ & $a_{33}$ & $A_{3}$ \\
\hline
\end{tabular}

There are some advantages to use priority relation ranking method to complete the ranking of the research objects in multi-attribute decision problems. First of all, we can use Step 2 for the first 
screening when there are many research objects, so that the ideal objects can be quickly selected. Second, the first screening is carried out on the basis of 0-degree risk value, so that the grades of the research objects that are left will no less than the grade threshold under each attribute. What's more, this method takes into account the overall situation. That is to say we take the lowest grade of the same research object under different attributes as the grade of the research object to participate in the first screening. It can not only ensure that the research object can meet the decision maker's requirements under each attribute, but also effectively avoid that the value of the good and bad attributes to be canceled out each other.

Example 8. It is assumed that a city's evaluation agency will conduct an overall assessment of 10 universities in the city. Top three universities will be selected as model universities, and the grades of the top three universities can't less than 2 of each attribute. The evaluation agency will give a comprehensive ranking of the three universities. The 10 universities in the city denoted by $U=\left\{x_{1}, x_{2}, \cdots, x_{10}\right\} . E=\left\{e_{1}, e_{2}, e_{3}, e_{4}\right\}$, and the parameters stand for "talents cultivation", "teaching staff", "scientific research level" and "school influence". The experts evaluate the 10 universities in the city and give their opinions denoted by $(N V, K)$ and we can see it in Table 17.

First of all, according to the Table 17 ( the $k$-degree risk value is 0 ), we can get the grades based on $(N V, K)$ denoted by Table 18. If $g_{\text {imin }}<2$, we will delete $x_{i}, i=1,2, \cdots, 10$. The decision maker determines the 0.5 -degree risk value based on the question type and his own risk preference. So a new neutrosophic vague N-soft set $(N V, K)^{\prime}$ is represented by Table 19.

Next, compute score function $S_{i j}^{\prime}$ denoted by Table 20 and compute the comparison table for $g_{i i^{*}}^{\prime}$ and $S_{i i^{*}}^{\prime}$ based on $(N V, K)^{\prime}$, as shown in Table 21.

What's more, we use priority relation ranking method to complete the ranking of the universities. In Table 21 , we find $\Delta_{2}=\max \left\{\Delta_{\text {imin }}\right\}=<1 ; 0.05>$, we will remove $x_{2}$ and consider $x_{2}$ is the first superior object under attribute $e_{1}$. If we go on in turn, we can get the orders of all the research objects under the same attribute $e_{1}$ as displayed in Tables 22-24. Hence, we known $x_{2} \succ x_{1} \succ x_{3} \succ x_{9} \succ x_{7}$ under the same attribute $e_{1}$.

Table 17. Tabular representation of the $(N V, K)$ in Example 8.

\begin{tabular}{ccc}
\hline$(N V, K)$ & $e_{1}$ & $e_{2}$ \\
\hline$x_{1}$ & $<3,[0.60,0.90],[0.10,0.40],[0.10,0.40]>$ & $<2,[0.40,0.70],[0.20,0.80],[0.30,0.60]>$ \\
$x_{2}$ & $<3,[0.70,0.90],[0.20,0.40],[0.10,0.30]>$ & $<3,[0.70,0.90],[0.20,0.30],[0.10,0.30]>$ \\
$x_{3}$ & $<3,[0.50,0.80],[0.10,0.30],[0.20,0.50]>$ & $<3,[0.60,0.70],[0.20,0.40],[0.30,0.40]>$ \\
$x_{4}$ & $<2,[0.30,0.80],[0.70,0.90],[0.20,0.70]>$ & $<3,[0.50,0.70],[0.20,0.80],[0.30,0.50]>$ \\
$x_{5}$ & $<1,[0.20,0.30],[0.60,0.70],[0.70,0.80]>$ & $<3,[0.70,0.80],[0.60,0.80],[0.20,0.30]>$ \\
$x_{6}$ & $<1,[0.20,0.40],[0.60,0.80],[0.60,0.80]>$ & $<2,[0.30,0.40],[0.50,0.70],[0.60,0.70]>$ \\
$x_{7}$ & $<2,[0.30,0.40],[0.50,0.60],[0.60,0.70]>$ & $<2,[0.40,0.50],[0.70,0.90],[0.50,0.60]>$ \\
$x_{8}$ & $<1,[0.20,0.30],[0.50,0.90],[0.70,0.80]>$ & $<4,[0.80,0.90],[0.20,0.30],[0.10,0.20]>$ \\
$x_{9}$ & $<3,[0.70,0.80],[0.30,0.70],[0.20,0.30]>$ & $<3,[0.50,0.70],[0.30,0.80],[0.30,0.50]>$ \\
$x_{10}$ & $<2,[0.30,0.50],[0.30,0.60],[0.50,0.70]>$ & $<3,[0.60,0.80],[0.50,0.80],[0.20,0.40]>$ \\
\hline$(N V, K)$ & \multicolumn{2}{c}{$e_{3}$} \\
\hline$x_{1}$ & $<3,[0.50,0.80],[0.10,0.90],[0.20,0.50]>$ & $<4,[0.80,0.90],[0.50,0.70],[0.10,0.20]>$ \\
$x_{2}$ & $<3,[0.70,0.90],[0.30,0.50],[0.10,0.30]>$ & $<3,[0.70,0.90],[0.10,0.20],[0.10,0.30]>$ \\
$x_{3}$ & $<4,[0.80,0.90],[0.30,0.50],[0.10,0.20]>$ & $<3,[0.60,0.80],[0.20,0.50],[0.20,0.40]>$ \\
$x_{4}$ & $<3,[0.50,0.60],[0.30,0.70],[0.40,0.50]>$ & $<1,[0.10,0.20],[0.80,0.90],[0.80,0.90]>$ \\
$x_{5}$ & $<3,[0.60,1.00],[0.30,0.50],[0.00,0.40]>$ & $<2,[0.40,0.60],[0.60,0.80],[0.40,0.60]>$ \\
$x_{6}$ & $<2,[0.30,0.40],[0.60,0.90],[0.60,0.70]>$ & $<3,[0.70,0.80],[0.40,0.50],[0.20,0.30]>$ \\
$x_{7}$ & $<4,[0.90,1.00],[0.10,0.20],[0.00,0.10]>$ & $<3,[0.70,0.90],[0.20,0.40],[0.10,0.30]>$ \\
$x_{8}$ & $<3,[0.60,0.70],[0.30,0.70],[0.30,0.40]>$ & $<1,[0.20,0.50],[0.50,0.70],[0.50,0.80]>$ \\
$x_{9}$ & $<2,[0.30,0.70],[0.20,0.50],[0.30,0.70]>$ & $<2,[0.30,0.40],[0.20,0.40],[0.60,0.70]>$ \\
$x_{10}$ & $<1,[0.20,0.80],[0.10,0.30],[0.20,0.80]>$ & $<4,[0.90,1.00],[0.30,0.40],[0.00,0.10]>$ \\
\hline & &
\end{tabular}


Table 18. The grades based on $(N V, K)$ in Example 8.

\begin{tabular}{cccccc}
\hline$(N V, K)$ & $\boldsymbol{e}_{\mathbf{1}}$ & $\boldsymbol{e}_{\mathbf{2}}$ & $\boldsymbol{e}_{\mathbf{3}}$ & $\boldsymbol{e}_{\mathbf{4}}$ & $g_{\text {imin }}$ \\
\hline$x_{1}$ & 3 & 2 & 3 & 4 & 2 \\
$x_{2}$ & 3 & 3 & 3 & 3 & 3 \\
$x_{3}$ & 3 & 3 & 4 & 3 & 3 \\
$x_{4}$ & 2 & 3 & 3 & 1 & 1 \\
$x_{5}$ & 1 & 3 & 3 & 2 & 1 \\
$x_{6}$ & 1 & 2 & 2 & 3 & 1 \\
$x_{7}$ & 2 & 2 & 4 & 3 & 2 \\
$x_{8}$ & 1 & 4 & 3 & 1 & 1 \\
$x_{9}$ & 3 & 3 & 2 & 2 & 2 \\
$x_{10}$ & 2 & 3 & 1 & 4 & 1 \\
\hline
\end{tabular}

Table 19. The new neutrosophic vague $N$-soft sets $(N V, K)^{\prime}$ in Example 8.

\begin{tabular}{ccc}
\hline$(N V, K)^{\prime}$ & $e_{1}$ & $e_{2}$ \\
\hline$x_{1}$ & $<3,[0.60,0.90],[0.10,0.40],[0.10,0.40]>$ & $<3,[0.40,0.70],[0.20,0.80],[0.30,0.60]>$ \\
$x_{2}$ & $<4,[0.70,0.90],[0.20,0.40],[0.10,0.30]>$ & $<4,[0.70,0.90],[0.20,0.30],[0.10,0.30]>$ \\
$x_{3}$ & $<3,[0.50,0.80],[0.10,0.30],[0.20,0.50]>$ & $<3,[0.60,0.70],[0.20,0.40],[0.30,0.40]>$ \\
$x_{7}$ & $<2,[0.30,0.40],[0.50,0.60],[0.60,0.70]>$ & $<2,[0.40,0.50],[0.70,0.90],[0.50,0.60]>$ \\
$x_{9}$ & $<3,[0.70,0.80],[0.30,0.70],[0.20,0.30]>$ & $<3,[0.50,0.70],[0.30,0.80],[0.30,0.50]>$ \\
\hline$(N V, K)^{\prime}$ & \multicolumn{2}{c}{$\boldsymbol{e}_{3}$} \\
\hline$x_{1}$ & $<3,[0.50,0.80],[0.10,0.90],[0.20,0.50]>$ & $<4,[0.80,0.90],[0.50,0.70],[0.10,0.20]>$ \\
$x_{2}$ & $<4,[0.70,0.90],[0.30,0.50],[0.10,0.30]>$ & $<4,[0.70,0.90],[0.10,0.20],[0.10,0.30]>$ \\
$x_{3}$ & $<4,[0.80,0.90],[0.30,0.50],[0.10,0.20]>$ & $<3,[0.60,0.80],[0.20,0.50],[0.20,0.40]>$ \\
$x_{7}$ & $<4,[0.90,1.00],[0.10,0.20],[0.00,0.10]>$ & $<4,[0.70,0.90],[0.20,0.40],[0.10,0.30]>$ \\
$x_{9}$ & $<3,[0.30,0.70],[0.20,0.50],[0.30,0.70]>$ & $<2,[0.30,0.40],[0.20,0.40],[0.60,0.70]>$ \\
\hline
\end{tabular}

Table 20. The score function $S_{i j}^{\prime}$ based on $(N V, K)^{\prime}$ in Example 8.

\begin{tabular}{ccc}
\hline$(N V, K)^{\prime}$ & $e_{1}$ & $e_{2}$ \\
\hline$x_{1}$ & $<3,0.75,0.25,0.25 ; 0.25>$ & $<3,0.55,0.50,0.45 ;-0.40>$ \\
$x_{2}$ & $<4,0.80,0.30,0.20 ; 0.30>$ & $<4,0.80,0.25,0.20 ; 0.35>$ \\
$x_{3}$ & $<3,0.65,0.20,0.35 ; 0.10>$ & $<3,0.65,0.30,0.35 ; 0.00>$ \\
$x_{7}$ & $<2,0.35,0.55,0.65 ;-0.85>$ & $<2,0.45,0.80,0.55 ;-0.90>$ \\
$x_{9}$ & $<3,0.75,0.50,0.25 ; 0.00>$ & $<3,0.60,0.55,0.40 ;-0.35>$ \\
\hline$(N V, K)^{\prime}$ & $\boldsymbol{e}_{3}$ & $\boldsymbol{e}_{4}$ \\
\hline$x_{1}$ & $<3,0.65,0.50,0.35 ;-0.20>$ & $<4,0.85,0.60,0.15 ; 0.10>$ \\
$x_{2}$ & $<4,0.80,0.40,0.20 ; 0.20>$ & $<4,0.80,0.15,0.20 ; 0.45>$ \\
$x_{3}$ & $<4,0.85,0.40,0.15 ; 0.30>$ & $<3,0.70,0.35,0.30 ; 0.05>$ \\
$x_{7}$ & $<4,0.95,0.15,0.05 ; 0.75>$ & $<4,0.80,0.30,0.20 ; 0.30>$ \\
$x_{9}$ & $<3,0.50,0.35,0.50 ;-0.35>$ & $<2,0.35,0.30,0.65 ;-0.60>$ \\
\hline
\end{tabular}

Table 21. Comparison table for $\Delta g_{i i^{*}}^{\prime}$ and $\Delta S_{i i^{*}}^{\prime}$ based on $e_{1}$.

\begin{tabular}{ccccccc}
\hline & $x_{\mathbf{1}}$ & $x_{\mathbf{2}}$ & $x_{\mathbf{3}}$ & $x_{\mathbf{7}}$ & $x_{\mathbf{9}}$ & $\boldsymbol{\Delta}_{\text {imin }}$ \\
\hline$x_{1}$ & $<0 ; 0>$ & $<-1 ;-0.05>$ & $<0 ; 0.15>$ & $<1 ; 1.10>$ & $<0 ; 0.25>$ & $<-1 ;-0.05>$ \\
$x_{2}$ & $<1 ; 0.05>$ & $<0 ; 0>$ & $<1 ; 0.20>$ & $<2 ; 1.15>$ & $<1 ; 0.30>$ & $<1 ; 0.05>$ \\
$x_{3}$ & $<0 ;-0.15>$ & $<-1 ;-0.20>$ & $<0 ; 0>$ & $<1 ; 0.95>$ & $<0 ; 0.10>$ & $<-1 ;-0.20>$ \\
$x_{7}$ & $<-1 ;-1.10>$ & $<-2 ;-1.15>$ & $<-1 ;-0.95>$ & $<0 ; 0>$ & $<-1 ;-0.85>$ & $<-2 ;-1.15>$ \\
$x_{9}$ & $<0 ;-0.25>$ & $<-1 ;-0.30>$ & $<0 ;-0.10>$ & $<1 ; 0.85>$ & $<0 ; 0>$ & $<-1 ;-0.30>$ \\
\hline
\end{tabular}


Table 22. Comparison table for $\Delta g_{i i^{*}}^{\prime}$ and $\Delta S_{i i^{*}}^{\prime}$ based on $e_{1}$ without $x_{2}$.

\begin{tabular}{cccccc}
\hline & $x_{\mathbf{1}}$ & $x_{\mathbf{3}}$ & $x_{\mathbf{7}}$ & $x_{\mathbf{9}}$ & $\boldsymbol{\Delta}_{\text {imin }}$ \\
\hline$x_{1}$ & $<0 ; 0>$ & $<0 ; 0.15>$ & $<1 ; 1.10>$ & $<0 ; 0.25>$ & $<0 ; 0.15>$ \\
$x_{3}$ & $<0 ;-0.15>$ & $<0 ; 0>$ & $<1 ; 0.95>$ & $<0 ; 0.10>$ & $<0 ;-0.15>$ \\
$x_{7}$ & $<-1 ;-1.10>$ & $<-1 ;-0.95>$ & $<0 ; 0>$ & $<-1 ;-0.85>$ & $<-1 ;-1.10>$ \\
$x_{9}$ & $<0 ;-0.25>$ & $<0 ;-0.10>$ & $<1 ; 0.85>$ & $<0 ; 0>$ & $<0 ;-0.25>$ \\
\hline
\end{tabular}

Table 23. Comparison table for $\Delta g_{i i^{*}}^{\prime}$ and $\Delta S_{i i^{*}}^{\prime}$ based on $e_{1}$ without $x_{2}$ and $x_{1}$.

\begin{tabular}{ccccc}
\hline & $x_{3}$ & $x_{7}$ & $x_{9}$ & $\boldsymbol{\Delta}_{\text {imin }}$ \\
\hline$x_{3}$ & $<0 ; 0>$ & $<1 ; 0.95>$ & $<0 ; 0.10>$ & $<0 ; 0.10>$ \\
$x_{7}$ & $<-1 ;-0.95>$ & $<0 ; 0>$ & $<-1 ;-0.85>$ & $<-1 ;-0.95>$ \\
$x_{9}$ & $<0 ;-0.10>$ & $<1 ; 0.85>$ & $<0 ; 0>$ & $<0 ;-0.10>$ \\
\hline
\end{tabular}

Table 24. Comparison table for $\Delta g_{i i^{*}}^{\prime}$ and $\Delta S_{i i^{*}}^{\prime}$ based on $e_{1}$ without $x_{2}, x_{1}$ and $x_{3}$.

\begin{tabular}{cccc}
\hline & $x_{7}$ & $x_{9}$ & $\Delta_{\text {imin }}$ \\
\hline$x_{7}$ & $<0 ; 0>$ & $<-1 ;-0.85>$ & $<-1 ;-0.85>$ \\
$x_{9}$ & $<1 ; 0.85>$ & $<0 ; 0>$ & $<1 ; 0.85>$ \\
\hline
\end{tabular}

Similarly, we can get $x_{2} \succ x_{3} \succ x_{9} \succ x_{1} \succ x_{7}$ under the same attribute $e_{2} ; x_{7} \succ x_{3} \succ x_{2} \succ x_{1} \succ x_{9}$ under the same attribute $e_{3} ; x_{2} \succ x_{7} \succ x_{1} \succ x_{3} \succ x_{9}$ under the same attribute $e_{4}$.

Finally, compute the rankings of the research objects under all attributes. Suppose the decision maker assigns weights to each attribute, $\omega_{1}=0.2, \omega_{2}=0.3, \omega_{3}=0.1, \omega_{4}=0.4$. And we can get $A_{9}>A_{7}>A_{1}>A_{3}>A_{2}$ from Table 25, we consider that $x_{2}$ is the first superior object, $x_{3}$ is the second superior object and $x_{1}$ is the third superior object under the $E$. Therefore, they will be selected as model universities.

Table 25. The ranking of the objects under all attributes.

\begin{tabular}{cccccc}
\hline & $\boldsymbol{e}_{\mathbf{1}} \cdot \mathbf{0 . 2}$ & $\boldsymbol{e}_{\mathbf{2}} \cdot \mathbf{0 . 3}$ & $\boldsymbol{e}_{\mathbf{3}} \cdot \mathbf{0 . 1}$ & $\boldsymbol{e}_{\mathbf{4}} \cdot \mathbf{0 . 4}$ & $\boldsymbol{A}_{\boldsymbol{i}}$ \\
\hline$x_{1}$ & 2 & 4 & 4 & 3 & $A_{1}=3.2$ \\
$x_{2}$ & 1 & 1 & 3 & 1 & $A_{2}=1.2$ \\
$x_{3}$ & 3 & 2 & 2 & 4 & $A_{3}=3.0$ \\
$x_{7}$ & 5 & 5 & 1 & 2 & $A_{7}=3.4$ \\
$x_{9}$ & 4 & 3 & 5 & 5 & $A_{9}=4.2$ \\
\hline
\end{tabular}

\subsection{Comparison Analysis}

In this paper, we presented neutrosophic vague $N$-soft sets based on both neutrosophic vague soft sets and $N$-soft sets. Many experts give different decision making methods, such as Akram et al. [23] used score function method and comparison table method to solve practical problems about $(F, N)$-soft sets.

Next, we applied score function method and comparison table method to Example 8. From Table 17, we can use the score function $S_{i j}^{\prime}=T_{i j}^{-}-I_{i j}^{-}-F_{i j}^{-}$to get score in Table 26. So we can get $x_{2} \succ x_{3} \succ x_{1} \succ$ $x_{7} \succ x_{9}$ from Table 27. The result of comparison table method is listed in Tables 28 and 29, so that we consider that $x_{2}$ is the first superior object, $x_{3}$ is the second superior object and $x_{1}$ is the third superior object under the $E$. 
Table 26. The score function $S_{i j}^{\prime}$ based on $(N V, K)$ in Example 8.

\begin{tabular}{ccc}
\hline$(N V, K)$ & $\boldsymbol{e}_{\mathbf{1}}$ & $\boldsymbol{e}_{\mathbf{2}}$ \\
\hline$x_{1}$ & $<3,0.60,0.10,0.10 ; 0.40>$ & $<2,0.40,0.20,0.30 ;-0.10>$ \\
$x_{2}$ & $<3,0.70,0.20,0.10 ; 0.40>$ & $<3,0.70,0.20,0.10 ; 0.40>$ \\
$x_{3}$ & $<3,0.50,0.10,0.20 ; 0.20>$ & $<3,0.60,0.20,0.30 ; 0.10>$ \\
$x_{7}$ & $<2,0.30,0.50,0.60 ;-0.80>$ & $<2,0.40,0.70,0.50 ;-0.80>$ \\
$x_{9}$ & $<3,0.70,0.30,0.20 ; 0.20>$ & $<3,0.50,0.30,0.30 ;-0.10>$ \\
\hline$(N V, K)$ & $\boldsymbol{e}_{3}$ & $\boldsymbol{e}_{\mathbf{4}}$ \\
\hline$x_{1}$ & $<3,0.50,0.10,0.20 ; 0.20>$ & $<4,0.80,0.50,0.10 ; 0.20>$ \\
$x_{2}$ & $<3,0.70,0.30,0.10 ; 0.30>$ & $<3,0.70,0.10,0.10 ; 0.50>$ \\
$x_{3}$ & $<4,0.80,0.30,0.10 ; 0.40>$ & $<3,0.60,0.20,0.20 ; 0.20>$ \\
$x_{7}$ & $<4,0.90,0.10,0.00 ; 0.80>$ & $<3,0.70,0.20,0.10 ; 0.40>$ \\
$x_{9}$ & $<2,0.30,0.20,0.30 ;-0.20>$ & $<2,0.30,0.20,0.60 ;-0.50>$ \\
\hline
\end{tabular}

Table 27. The total score based on $(N V, K)$ in Example 8.

\begin{tabular}{cccccc}
\hline$(N V, K)$ & $\boldsymbol{e}_{\mathbf{1}}$ & $\boldsymbol{e}_{\mathbf{2}}$ & $\boldsymbol{e}_{\mathbf{3}}$ & $\boldsymbol{e}_{\mathbf{4}}$ & The Total Score \\
\hline$x_{1}$ & $<3,0.40>$ & $<2,-0.10>$ & $<3,0.20>$ & $<4,0.20>$ & $<12,0.70>$ \\
$x_{2}$ & $<3,0.40>$ & $<3,0.40>$ & $<3,0.30>$ & $<3,0.50>$ & $<12,1.60>$ \\
$x_{3}$ & $<3,0.20>$ & $<3,0.10>$ & $<4,0.40>$ & $<3,0.20>$ & $<13,0.90>$ \\
$x_{7}$ & $<2,-0.80>$ & $<2,-0.80>$ & $<4,0.80>$ & $<3,0.40>$ & $<11,-0.40>$ \\
$x_{9}$ & $<3,0.20>$ & $<3,-0.10>$ & $<2,-0.20>$ & $<2,-0.50>$ & $<10,-0.60>$ \\
\hline
\end{tabular}

By comparing the ranking results of the existing methods and our proposed method, we observe that their result is the same, which means that there is a consistency among our proposed decision making method and the existing methods. But the method we proposed is more reasonable because the idea of probability is implied in the grade. Therefore, the presented method is more reasonable for solving practical decision application problems. In our daily life, the decision maker can use different methods according to their preferences.

Table 28. Comparison table for $S_{i j}^{\prime}$.

\begin{tabular}{cccccc}
\hline & $x_{\mathbf{1}}$ & $x_{\mathbf{2}}$ & $x_{\mathbf{3}}$ & $x_{\mathbf{7}}$ & $x_{\mathbf{9}}$ \\
\hline$x_{1}$ & 4 & 1 & 2 & 2 & 4 \\
$x_{2}$ & 1 & 4 & 3 & 3 & 4 \\
$x_{3}$ & 3 & 2 & 4 & 2 & 4 \\
$x_{7}$ & 2 & 1 & 2 & 4 & 2 \\
$x_{9}$ & 1 & 0 & 1 & 2 & 4 \\
\hline
\end{tabular}

Table 29. The result of comparison score table.

\begin{tabular}{ccccc}
\hline & Grade Sum (g) & Row Sum (r) & Column Sum (c) & Final Sum (r-c) \\
\hline$x_{1}$ & 12 & 13 & 11 & 2 \\
$x_{2}$ & 12 & 15 & 8 & 7 \\
$x_{3}$ & 13 & 15 & 12 & 3 \\
$x_{7}$ & 11 & 11 & 13 & -2 \\
$x_{9}$ & 10 & 8 & 18 & -10 \\
\hline
\end{tabular}

\section{Conclusions}

In this paper, neutrosophic vague $N$-soft sets by a suitable combination of neutrosophic vague sets with $\mathrm{N}$-soft sets is proposed for the first time. The novel hybrid model combines the advantages of existing models. We use $\delta_{k p}(x)$ containing the idea of probability to define the grade of object firstly. It will not ignore the effect of hesitant parts and false membership in dealing with vague and uncertain 
problems. Some useful operations and propositions are given. Moreover, the priority relation ranking method based on neutrosophic vague $N$-soft sets is introduced for the first time. By comparing the ranking results of the existing methods and our proposed method, we observe that their result is the same, which means that there is a consistency among our proposed decision making method and the existing methods. But the method we proposed is more reasonable because the idea of probability is implied in the grade. Finally, the method is used in practical multi-attribute decision making problems.

The method mentioned in this article can not only select the best research object, but also sort the research objects. If there are many research objects, when sorting the research objects, although the results are more reliable, the calculation is relatively complicated. Of course, if it can be translated into a computer language, it will save a lot of time. In the era of big data, there will be more and more cases to prove the effectiveness and flexibility of this method. The different hybrid models provide more accurate and better choices. In the future, we can try to build richer connections between the between $\mathrm{N}$-soft sets and other soft computing models.

Author Contributions: Conceptualization, J.L. and Y.C.; methodology, Y.C.; validation, Y.C., Z.C. and Y.Z.; writing-original draft preparation, Y.C.; writing-review and editing, J.L., Y.C., Z.C. and Y.Z.; funding acquisition, J.L. All authors have read and agreed to the published version of the manuscript.

Funding: This work is partially supported by Nature Science Foundation of Hebei Province under Grant No. F2014501046.

Conflicts of Interest: The authors declare no conflict of interest.

\section{References}

1. Zadeh, L.A. Fuzzy sets. Inf. Control 1965, 8, 338-353. [CrossRef]

2. Pawlak, Z. Rough sets. Int. J. Parallel Program. 1982, 11, 341-356. [CrossRef]

3. Atanassov, K.T. Intuitionistic fuzzy sets. Fuzzy Sets Syst. 1986, 20, 87-96. [CrossRef]

4. Gau, W.L.; Buehrer, D.J. Vague sets. IEEE Trans. Syst. Man Cybern. 1993, 23, 610-614. [CrossRef]

5. Molodtsov, D. Soft set theory-first results. Comput. Math. Appl. 1999, 37, 19-31. [CrossRef]

6. Maji, P.K.; Biswas, R.; Roy, A.R. Fuzzy soft sets. Fuzzy Math 2001, 9, 589-602.

7. Maji, P.K.; Biswas, R.; Roy, A.R. Intuitionistic fuzzy soft sets. Fuzzy Math 2001, 9, 677-692.

8. Yang, X.; Lin, T.Y.; Yang, J.; Li, Y.; Yu, D. Combination of interval-valued fuzzy set and soft set. Comput. Math. Appl. 2009, 58, 521-527. [CrossRef]

9. Xu, W.; Ma, J.; Wang, S.; Hao, G. Vague soft sets and their properties. Comput. Math. Appl. 2010, 58, 521-527. [CrossRef]

10. Alkhazaleh, S.; Salleh, A.R.; Hassan, N. Possibility fuzzy soft set. Adv. Decis. Sci. 2011.10.1155/2011/479756. [CrossRef]

11. Feng, F. Research on Uncertain Theory Fusion Based on Soft Set; Shanxi Normal University: Xi'an, China, 2012.

12. Peng, X.D.; Yang, Y. Approaches to interval-valued intuitionistic hesitant fuzzy soft sets based decision making. Fuzzy Math Inf. 2015, 10, 657-680.

13. Smarandache, F. Neutrosophic set-A generalisation of the intuitionistic fuzzy sets. Int. J. Pure Appl. Math. 2005, 24, 287-297.

14. Maji, P.K. Neutrosophic soft set. Ann. Fuzzy Math. Inform. 2013, 5, 157-168.

15. Alkhazaleh, S. Neutrosophic vague set theory. Crit. Rev. 2015.10.5281/zenodo.22602. [CrossRef]

16. Said, B.; Smarandache, F. Intuitionistic neutrosophic soft set. J. Inf. Comput. Sci. 2013, 8, 130-140.

17. Deli, I.; Çağman, N. Interval-valued neutrosophic soft sets and its decision making. Int. J. Mach. Learn. Cybern. 2014. [CrossRef]

18. Ashraf, Al.Q.; Hassan, N. Neutrosophic Vague Soft Set and its Applications. Malays. J. Math. Sci. 2017, 11, $141-163$.

19. Ye, J. Similarity measures between interval neutrosophic sets and their applications in multicriteria decision-making. J. Intell. Fuzzy Syst. 2014, 26, 165-172. [CrossRef]

20. Şahin, R.; Küçük, A. On similarity and entropy of neutrosophic soft sets. J. Intell. Fuzzy Syst. 2014, 27, 2417-2430. [CrossRef] 
21. Ye, J. Improved correlation coefficients of single valued neutrosophic sets and interval neutrosophic sets for multiple attribute decision making. J. Intell. Fuzzy Syst. 2014, 27, 2453-2462. [CrossRef]

22. Fatimah, F.; Rosadi, D.; Hakim, R.B.F.; Alcantud, J.C.R. N-soft sets and their decision-making algorithms. Soft Comput. 2018, 22, 3829-3842. [CrossRef]

23. Akram, M.; Adeel, A.; Alcantud, J.C.R. Fuzzy N-soft sets: a novel model with applications. Intell. Fuzzy Syst. 2018, 35, 4757-4771. [CrossRef]

24. Akram, M.; Adeel, A.; Alcantud, J.C.R. Group decision-making methods based on hesitant N-soft sets. Expert Syst. Appl. 2019, 115, 95-105. [CrossRef]

25. Akram, M.; Adeel, A.; Alcantud, J.C.R. Hesitant fuzzy N-soft sets: A new model with applications in decision-making. Intell. Fuzzy Syst. 2019, 36, 6113-6127. [CrossRef]

26. Akram, M.; Adeel, A. TOPSIS Approach for MAGDM Based on Interval-Valued Hesitant Fuzzy N-soft Environment. Int. J. Fuzzy Syst. 2019, 21, 993-1009. [CrossRef]

27. Akram, M.; Ali, G.; Alcantud, J.C.R. New decision-making hybrid model: intuitionistic fuzzy N-soft rough sets. Soft Comput. 2019, 23, 9853-9868. [CrossRef]

28. Garg, H.; Nancy. Multiple criteria decision making based on frank choquet heronian mean operator for single-valued neutrosophic sets. Appl. Comput. Math. 2019, 18, 163-188.

29. Liu, P.; Wang, Y.; Jia, F.; Fujita, H. A multiple attribute decision making three-way model for intuitionistic fuzzy numbers. Int. J. Approx. Reason. 2020. [CrossRef]

30. Ataei, Y.; Mahmoudi, A.; Feylizadeh, M.R.; Li, D.F. Ordinal Priority Approach (OPA) in Multiple Attribute Decision-Making. Appl. Soft Comput. J. 2020. [CrossRef]

31. Yu, G.; Zeng, S.; Zhang, C. Single-Valued Neutrosophic Linguistic-Induced Aggregation Distance Measures and Their Application in Investment Multiple Attribute Group Decision Making. Symmetry 2020, 12, 207. [CrossRef]

32. Wang, H.; Smarandache, F.; Zhang, Y. Single valued neutrosophic sets. Multispace Multistruct. 2010, 4, 410-413.

33. Feng, F.; Fujita, H.; Ali, M.I.; Yager R.R.; Liu, X. Another view on generalized intuitionistic fuzzy soft sets and related multi-attribute decision making methods. IEEE Trans. Fuzzy Syst. 2018, 27, 474-488. [CrossRef]

(C) 2020 by the authors. Licensee MDPI, Basel, Switzerland. This article is an open access article distributed under the terms and conditions of the Creative Commons Attribution (CC BY) license (http:/ / creativecommons.org/licenses/by/4.0/). 